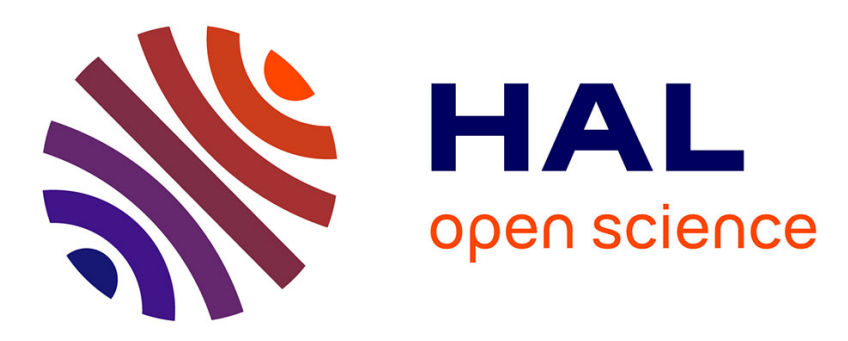

\title{
Characterization of geological boundaries using 1-D wavelet transform on gravity data: Theory and application to the Himalayas
}

\author{
Guillaume Martelet, P Sailhac, F Moreau, M Diament
}

\section{To cite this version:}

Guillaume Martelet, P Sailhac, F Moreau, M Diament. Characterization of geological boundaries using 1-D wavelet transform on gravity data: Theory and application to the Himalayas. Geophysics, 2000, 66, pp.1116-1129. 10.1190/1.1487060 . insu-01354820

HAL Id: insu-01354820

https://hal-insu.archives-ouvertes.fr/insu-01354820

Submitted on 19 Aug 2016

HAL is a multi-disciplinary open access archive for the deposit and dissemination of scientific research documents, whether they are published or not. The documents may come from teaching and research institutions in France or abroad, or from public or private research centers.
L'archive ouverte pluridisciplinaire HAL, est destinée au dépôt et à la diffusion de documents scientifiques de niveau recherche, publiés ou non, émanant des établissements d'enseignement et de recherche français ou étrangers, des laboratoires publics ou privés. 


\title{
Characterization of geological boundaries using 1-D wavelet transform on gravity data: Theory and application to the Himalayas
}

\author{
G. Martelet*, P. Sailhac*, F. Moreau ${ }^{\ddagger}$, and M. Diament*
}

\begin{abstract}
We investigate the use of the continuous wavelet transform for gravity inversion. The wavelet transform operator has recently been introduced in the domain of potential fields both as a filtering and a source-analysis tool. Here we develop an inverse scheme in the wavelet domain, designed to recover the geometric characteristics of density heterogeneities described by simple-shaped sources. The 1-D analyzing wavelet we use associates the upward continuation operator and linear combinations of derivatives of any order. In the gravity case, we first demonstrate how to localize causative sources using simple geometric constructions. Both the upper part of the source and the whole source can be studied when considering low or high altitudes, respectively. The homogeneity degree of the source is deduced without prior information and allows us to infer its shape. Introducing complex wavelets, we derive analytically the scaling behavior of the wavelet coefficients for the dyke and the step sources. The modulus term is used in an inversion procedure to recover the thickness of the source. The phase term provides its dip. This analysis is performed on gravity data we measured along a profile across the Himalayas in Nepal. Good agreement of our results with well-documented thrusting structures demonstrates the applicability of the method to real data. Also, deeper, less constrained structures are characterized.
\end{abstract}

\section{INTRODUCTION}

One of the most common problems encountered in geophysical studies is how to determine the geometry of geological contacts at depth. To address this problem, we use the wavelet transform applied to gravity, an operator recently introduced in the potential-fields domain by Moreau (1995). To better understand the specific characteristics of this method, we first review existing techniques and the role they have taken in building modern investigation methods in the domain of potential fields. Generally, geophysical modeling is addressed in terms of depth to simple-shaped sources or depth to geometric interfaces that represent geological contacts. The latter approach has led to a variety of different inversion techniques that are all subject to the well-known nonuniqueness of potential-fields causative sources. Solving this problem always requires additional a priori information. On the other hand, several techniques transform the measured field and characterize the sources responsible for it. Empirical graphical procedures were first proposed. They allow reasonable determinations of the depth to magnetization distributions of defined shapes (Peters, 1949). The next stage was devoted to comparing an observed field to analytic models-first, by means of charts (e.g., Chastenet de Gery and Naudy, 1957), and later by a variety of different techniques that use synthetic model fitting, such as Werner deconvolution (e.g., Werner, 1953; Hartman et al., 1971), Euler deconvolution (e.g., Thompson, 1982; Reid et al., 1990), and analytic signal (e.g., Nabighian, 1972; Roest et al., 1992). Extensive applications and improvements of these methods have been published, such as the use of vertical gradients (e.g., Marson and Klingele, 1993; Debeglia and Corpel, 1997) and considering the effect of noise in data (Keating, 1998). These techniques have almost always been used assuming a chosen geometry of the source. But very recently, Huang (1996), Stavrev (1997), and Barbosa et al. (1999) have investigated the recovery of both shape and depth of sources using methods derived from the Euler deconvolution, as do Hsu et al. (1998) by adapting the analytic signal technique. In our paper, using the wavelets formalism applied to gravity, we propose an inversion scheme that lets us simultaneously estimate the depth and shape of the source as well as its dip and thickness.

Another recognized family of methods developed since the late Sixties for the recovery of causative sources of potential fields focuses on the scaling properties of the field. Fourier analysis has triggered a wide range of applications in the potential-fields domain, both for data filtering (Bhattacharya,

Manuscript received by the Editor August 9, 1999; revised manuscript received April 24, 2000.

*Institut de Physique du Globe, Laboratoire de Gravimétrie et Géodynamique, UMR 7577, case 89, 4 place Jussieu, 75252 Paris, France.

E-mail: g.martelet@brgm.fr; sailhac@rhodo.u-strasbg.fr; diament@ipgp.jussieu.fr.

†Campus Scientifique de Beaulieu, Geosciences Rennes, Bat. 15, 263 av. du General Leclerc, CS 74205, 35042 Rennes, France.

(C) 2001 Society of Exploration Geophysicists. All rights reserved. 
1972) and transforming and for sources characterization [see Blakely (1995) for a more thorough review]. Recently, the wavelet transform has been developed as a powerful analysis tool (Holschneider, 1995) and has been introduced in the geophysical domain [Foufoula-Georgiou and Kumar (1994) and references therein]. The purpose of both Fourier and wavelet operators is to investigate the behavior of the measured field in terms of wavelengths. The Fourier technique is efficient at indicating depth to stationary undulating surfaces (Spector and Grant, 1970), but its application may become a problem when the signal is strongly nonstationary. The use of the wavelet operator instead, as first defined by Grossman and Morlet (1984), allows wavelength-adaptive convolution operators that vary with the wavelength of the studied portion of signal. In other words, the wavelet operator can focus on individual objects. Moreover, the special class of wavelets we use in this study lets us analyze the scaling properties of the source via upward continuation. A general $n$-dimensional theoretical framework for local homogeneous sources has been developed by Moreau et al. (1997, 1999). With this approach, Sailhac et al. (2000) address the magnetic anomalies interpretation. Here, we discuss the wavelet technique capabilities for gravity data. We briefly recall the basic principles of wavelet transform theory. Then we derive analytic expressions of the wavelet coefficients for simple-shaped gravity sources and test the method on several synthetic examples. We show that horizontal and vertical location of the source as well as its shape, vertical extension, and dip can be estimated. Finally, we analyze a real gravity anomaly profile measured across the Himalayan range in Nepal.

\section{FUNDAMENTALS OF CONTINUOUS WAVELETS FOR POTENTIAL FIELDS}

In the potential-fields domain, the use of orthonormal wavelets has proven helpful for filtering (Fedi and Quarta, 1998; Ridsdill-Smith and Dentith, 1999; Ridsdill-Smith et al., 1999). For analysis purposes, Moreau et al. (1997) introduce a special basis of continuous wavelets derived from a Poisson semigroup kernel. This new type of wavelet decomposition is specifically well suited for the study of potential fields since it acts on this type of data as the upward continuation operator does. Furthermore, it utilizes vertical and horizontal derivatives of the field. This induces special geometric properties that have been investigated for magnetic anomalies, in the case of horizontal derivatives only (Hornby et al., 1999) or for both horizontal and vertical derivatives and either in the real domain (Moreau et al., 1999) or in the complex domain (Sailhac et al., 2000). The association of derivation and upward continuation in a single operator via the wavelet formalism is interesting since useful properties of both operators are combined. Indeed, the use of derivatives of the field ensures a better outlining of anomalies (Cordell and Grauch, 1985; Marson and Klingele, 1993; Debeglia and Corpel, 1997; Pilkingston, 1997). In addition, derivatives can be associated linearly to obtain special operators such as the analytic signal (e.g., Nabighian, 1972). Also, as suggested by Paul et al. (1966), the knowledge of the field at several altitudes (or dilations) gives valuable information concerning its scaling properties. To a certain extent, this can be related to the study of gradient tensors (e.g., Nelson, 1988; Pedersen and Rasmussen, 1990). This last specificity of the wavelet transform is particularly attractive since (1) it permits study of the properties of both the upper part and the center of the source, depending on the range of dilations considered, and (2) when considering the domain of high dilations, it permits study of extended sources and reduces the effect of noise. Indeed, when an extended source is considered from the high dilations, it behaves like a local one. This asymptotic behavior allows extension of the application of the theory developed for local homogeneous sources (Moreau et al., 1997). Also, the higher $\mathrm{S} / \mathrm{N}$ ratio along the extrema lines allows a reasonably accurate study even when the data are noisy (Moreau et al., 1999).

\section{CONTINUOUS WAVELET THEORY: APPLICATION TO THE GRAVITY FIELD}

\section{The Cauchy wavelet and local sources}

We briefly recall the basic theory developed by Moreau et al. $(1997,1999)$, which we apply to the case of a 2-D physical space $(x, a)$ where $x$ is the abscissa along the studied profile and $a$ is the dilation (positive upward). The continuous wavelet transform $\mathcal{W}$ of a function $\phi_{0}(x)$ is defined as a convolution product

$$
\begin{aligned}
\mathcal{W}_{\psi \mid \phi_{0}}(b, a) & =\int_{-\infty}^{+\infty} \frac{d x}{a} \psi\left(\frac{b-x}{a}\right) \phi_{0}(x) \\
& =\left(\mathcal{D}_{a} \psi * \phi_{0}\right)(b),
\end{aligned}
$$

where $\psi$ is the analyzing wavelet, $b$ is a position parameter, and the dilation operator $\mathcal{D}_{a}$ is defined by

$$
\mathcal{D}_{a} \psi(x)=\frac{1}{a} \psi\left(\frac{x}{a}\right) .
$$

Moreau et al. (1997, 1999) show that a special class of wavelets $\psi^{a}$ is obtained when a Fourier multiplier homogeneous of degree $\gamma$ (equivalent to a derivation of order $\gamma$ ) and a dilation are applied to the Poisson semigroup kernel. This kernel actually defines the well-known upward continuation filter $P_{a}(x)$, which transforms the harmonic field $\phi(., z)$ from measured level $z$ to the level $z+a$ (e.g., Bhattacharyya, 1972):

$$
P_{a}(x)=\frac{1}{\pi} \frac{a}{a^{2}+x^{2}},
$$

where the notation $\phi(., z)$ means that the operator applied to the field $\phi(x, z)$ only acts on the variable $x$, while $z$ remains unchanged. Two typical real wavelets can then be considered: the horizontal $\psi_{x}$ and the vertical $\psi_{z}$. They correspond, respectively, to one horizontal or one vertical derivation of $P_{1}$, that is, the first horizontal or vertical derivative upward-continued at level $a=1$. Wavelets of higher order of derivation $\gamma$ are obtained by $\gamma-1$ additional derivations along the $x$-axis only. Their expressions in space and Fourier domain are, respectively,

$$
\begin{aligned}
& \left\{\begin{array}{l}
\psi_{x}^{\gamma}(x)=\partial_{x}^{\gamma} P_{1}(x) \\
\psi_{z}^{\gamma}(x)=\left.\partial_{x}^{\gamma-1} \partial_{a} P_{a}(x)\right|_{a=1}
\end{array}\right. \\
& \left\{\begin{array}{l}
\hat{\psi}_{x}^{\gamma}(u)=(i 2 \pi u)^{\gamma} e^{-2 \pi|u|} \\
\hat{\psi}_{z}^{\gamma}(u)=(i 2 \pi u)^{\gamma-1}(-2 \pi|u|) e^{-2 \pi|u|}
\end{array}\right.
\end{aligned}
$$

Finally, the $\gamma$-order wavelet transform of the potential field $\phi(x, z)$ measured at level $z$ is obtained at altitudes $a \geq 0$. It 
corresponds to the upward-continued derivatives of the field $\phi$, also known as wavelet coefficients:

$$
\mathcal{W}_{\psi_{x}^{\gamma} \mid \phi(., z)}(x, a)=a^{\gamma} \frac{\partial^{\gamma-1} \phi_{x}(x, z+a)}{\partial x^{\gamma-1}}
$$

and

$$
\mathcal{W}_{\psi_{z}^{\gamma} \mid \phi(., z)}(x, a)=a^{\gamma} \frac{\partial^{\gamma-1} \phi_{z}(x, z+a)}{\partial x^{\gamma-1}},
$$

where $\phi_{x}(., z)$ and $\phi_{z}(., z)$ are the horizontal and upward vertical derivatives of $\phi$ at level $z$. To these two real wavelets corresponds a complex wavelet (Moreau, 1995) defined as the sum of $\psi_{x}^{\gamma}$ for the real part and its Hilbert transform $\mathcal{H}\left[\psi_{x}^{\gamma}\right]=-\psi_{z}^{\gamma}$ for the imaginary part ( $z$ positive downward):

$$
\psi_{c}^{\gamma}=\psi_{x}^{\gamma}-i \psi_{z}^{\gamma} .
$$

Thus, the complex wavelet transform of the potential field $\phi(., z)$ is

$$
\mathcal{W}_{\psi_{c}^{\gamma} \mid \phi(., z)}(x, a)=\mathcal{W}_{\psi_{x}^{\gamma} \mid \phi(., z)}(x, a)-i \mathcal{W}_{\psi_{z}^{\gamma} \mid \phi(., z)}(x, a) .
$$

Real and imaginary parts of the complex wavelet transform are, respectively, the horizontal and vertical real wavelet coefficients. Expression (9) can be related to the modulus of the 1-D analytic signal (Nabighian, 1972) applied to the field $\phi(x, z)$ :

$$
|A(x)|=\sqrt{\left(\frac{\partial}{\partial x} \phi_{x}(x, z)\right)^{2}+\left(\frac{\partial}{\partial z} \phi_{z}(x, z)\right)^{2}} .
$$

Indeed, the modulus of the $\gamma=1$ complex wavelet transform represents the analytic signal that would have been computed at any level above the measurement level:

$$
\left|\mathcal{W}_{\psi_{c}^{1} \mid \phi(., z)}(x, a)\right| / a^{2}=\sqrt{\left[\phi_{x}(x, z+a)\right]^{2}+\left[\phi_{z}(x, z+a)\right]^{2}},
$$

where $\phi_{x}(., z)$ and $\phi_{z}(., z)$ are the horizontal and upward vertical derivatives of the potential $\phi(x, z)$. Similarly, from equations (6), (7), and (9), any order $\gamma$ of the wavelet transform can be obtained:

$$
\begin{aligned}
& \left(\frac{\left|\mathcal{W}_{\psi_{c}^{\gamma+1} \mid \phi(., z)}(x, a)\right|}{a^{\gamma+1}}\right)^{2} \\
& =\left(\frac{\partial^{\gamma}}{\partial x^{\gamma}} \phi_{x}(x, z+a)\right)^{2}+\left(\frac{\partial^{\gamma}}{\partial x^{\gamma}} \phi_{z}(x, z+a)\right)^{2} .
\end{aligned}
$$

\section{Geometric developments}

We now consider a gravity field $\phi(., z=0)$, i.e., measured at level $z=0$, which results from a local homogeneous source located at abscissa $x=0$ and depth $z=z_{0}$. The homogeneity $\alpha$ of the source can be stated via the scaling relation $\rho(\lambda x) \sim \lambda^{\alpha} \rho(x)$. This is not to be confused with the homogeneity $n$ of the field $T(x)$ introduced by Thompson (1982), $T(\lambda x) \sim \lambda^{n} T(x)$. The homogeneity of the source is a generalization of the homogeneity of the field. Both exponents $n$ and $\alpha$ are related via (Sailhac et al., 2000)

$$
n=\alpha+1,
$$

and $N=-n$ is a shape factor characteristic of the source, which is known as the Euler structural index. Under the assumption that the source is homogeneous, Moreau (1995) gives the relation that exists between the wavelet coefficients at two levels $a$ and $a^{\prime \prime}$ (for any of the $\psi_{x}^{\gamma}, \psi_{z}^{\gamma}$, or $\psi_{c}^{\gamma}$ wavelets):

$$
\begin{aligned}
\mathcal{W}_{\psi \gamma_{\mid \phi(., z=0)}}(x, a)= & \left(\frac{a}{a^{\prime \prime}}\right)^{\gamma}\left(\frac{a^{\prime \prime}+z_{0}}{a+z_{0}}\right)^{-\beta} \\
& \times \mathcal{W}_{\psi \gamma}{ }_{\mid \phi(., z=0)}\left(x \frac{a^{\prime \prime}+z_{0}}{a+z_{0}}, a^{\prime \prime}\right) .
\end{aligned}
$$

This relation exhibits a double scaling law with two exponent parameters; exponent $\gamma$ is the order of derivation of the wavelet, and exponent $\beta$ is associated to the homogeneity degree $\alpha$ of the source. On gravity profiles, the relation is given by

$$
\beta=\alpha+1-\gamma
$$

Combining equations (13) and (15) yields the relation between $\beta$ and Euler structural index $N$ :

$$
\beta=-(\gamma+N) .
$$

Equation (14) defines a set of lines in the $(x, a)$ space which satisfy $x /\left(z_{0}+a\right)=$ constant. If we name $k$ this constant, for various $k$ we obtain a family of lines that intersect at the location of the source $\left(x=0, z=z_{0}\right)$ inside the lower half-plane (Figure 1a). Equivalently, in three dimentions the wavelet transform exhibits a conelike structure pointing to the source. In practice, we only use the modulus maxima lines (on which the $\mathrm{S} / \mathrm{N}$ ratio is the best) to locate the source (see the example of the pointlike source in two dimensions in Figure 1). Similarly, when considering the complex wavelet coefficients, isovalues of their modulus also draw a cone pointing to the source. Once the depth $z_{0}$ of the source is obtained, exponent $\beta$ (related to the shape of the source) is computed as the slope of $\log \left(|\mathcal{W}| / a^{\gamma}\right)$ versus $\log \left(a+z_{0}\right)$ along modulus maxima lines of the wavelet transform. Therefore, at this point, with only the assumption
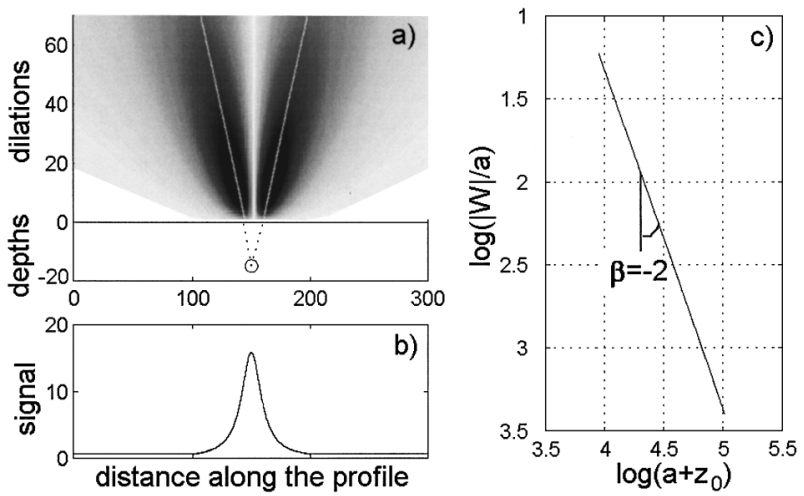

FIG. 1. (a)Wavelet transform coefficients of the gravity field [shown in (b)] in the case of a pointlike 2-D source. Derivation operator is $\partial / \partial x$. Maxima lines are superimposed in white; they converge toward the source. Along one such line [in (c)] in the case of the pointlike source, the scaling relation between $\log \left(\left|\mathcal{W}_{a}\right| / a^{\gamma}\right)$ and $\log (a+z)$ is strictly linear when $z=z_{0}$. The obtained slope $\beta=-2$ is directly related to the homogeneity degree of the source $\alpha=-2$ using equation (15) (see text for details). 
of homogeneity of the source, we are able to recover both the depth $z_{0}$ and the shape of a local source. Euler deconvolution or analytic signal methods cannot address both questions without additional information.

\section{STUDY OF SOME SYNTHETIC SOURCES}

Theory has been developed for local homogeneous sources for which equation (14) holds. For nonlocal or nonhomogeneous sources (at least for magnetic studies), the asymptotic behavior of the wavelet transform has proven to conveniently extend the domain of the technique's application (Moreau et al., 1999; Sailhac et al., 2000). To determine precisely the behavior of the wavelet operator in the gravity case, we derive the analytic expressions for several simple-shaped sources: vertical and inclined steps as well as vertical and inclined dykes. First we show that in the gravity case as well as for magnetics the theory developed for local homogeneous sources (Moreau et al., 1997) can be satisfactorily extrapolated to extended sources. In the case of extended sources, following the approach of Moreau et al. (1999), we show that the determination of average depth and shape factor can be improved greatly when focus on a certain range of dilations is done using a trialand-error process. Also in the case of the inclined step, using the phase term of complex wavelets, we propose two methods for determining the dip of the source. Finally, in addition to depth, shape, and dip, we show that under certain assumptions it is possible to recover the thickness of the extended step. This is achieved by inverting simultaneously the average depth $z_{0}$, the shape factor $\beta$, and the thickness $h$. The generalization to the case of the dyke source is also briefly described. In all cases, analytic expressions are determined for 2-D sources.

\section{The vertical step}

We consider a 2-D vertical step, i.e., of infinite length in a direction perpendicular to the profile. The step extends between $z_{1}=z_{0}-(h / 2)$ and $z_{2}=z_{0}+(h / 2)$ and has a constant density contrast, positive inside the step (see geometry and extended notations in Figure 2a).

We consider the complex wavelet transform which, as previously mentioned, corresponds to the upward-continued analytic signal. Analytic developments are reported in Appendix $\mathrm{A}$, in the general case of the inclined step. For the vertical step, the wavelet coefficients are

$$
\mathcal{W}_{\psi_{c}^{1} \mid g_{z}(., z=0)}(x, a)=G \rho a\left[\ln \left(\frac{R_{u}^{2}}{R_{0}^{2}}\right)+2 i\left(\phi_{0}-\phi_{u}\right)\right],
$$

with $G$ the universal gravitational constant, $\rho$ the density contrast, and $R_{0}, R_{u}, \phi_{0}$, and $\phi_{u}$ shown in Figure 2a. The associated modulus and phase are, respectively,

$$
\left|\mathcal{W}_{\psi_{c}^{1} \mid g_{z}(., z=0)}(x, a)\right|=G \rho a \sqrt{\left(2\left(\phi_{0}-\phi_{u}\right)\right)^{2}+2 \ln \left(\frac{R_{u}^{2}}{R_{0}^{2}}\right)}
$$

and

$$
\Phi_{\psi_{c}^{1} \mid g_{z}(., z=0)}(x, a)=\tan ^{-1}\left(\frac{2\left(\phi_{0}-\phi_{u}\right)}{\ln \left(\frac{R_{u}^{2}}{R_{0}^{2}}\right)}\right) .
$$

As described for the analytic signal (Nabighian, 1972), the modulus has a unique maximum located at the vertical contact $\left(x=x_{0}\right)$. In our case, this maximum turns into a straight modulus maxima line that runs across the scales (see Figure $3 a$ ). The phase along the modulus maxima line is 0 . Therefore, along the maxima line, equation (17) yields

$$
\mathcal{W}_{\psi_{c}^{1} \mid g_{z}(., z=0)}(X=0, a)=G \rho a \ln \left[\frac{\left(z_{0}+a+h / 2\right)^{2}}{\left(z_{0}+a-h / 2\right)^{2}}\right] .
$$

When $z_{0}+a \gg h / 2$ - that is, when considering high dilations, deep sources, or thin sources-asymptotic behavior of the wavelet coefficients can be stated via a Taylor expansion (this equation is derived in the general case of the inclined step in Appendix B):

$$
\begin{gathered}
\left|\mathcal{W}_{\psi_{c}^{1} \mid g_{z}(., z=0)}(X=0, a)\right|=4 G \rho a\left(\frac{h / 2}{z_{0}+a}\right) \\
\times\left[1+\frac{1}{3}\left(\frac{h / 2}{z_{0}+a}\right)^{2}\right]+O\left(\frac{h / 2}{z_{0}+a}\right)^{5} .
\end{gathered}
$$

a)

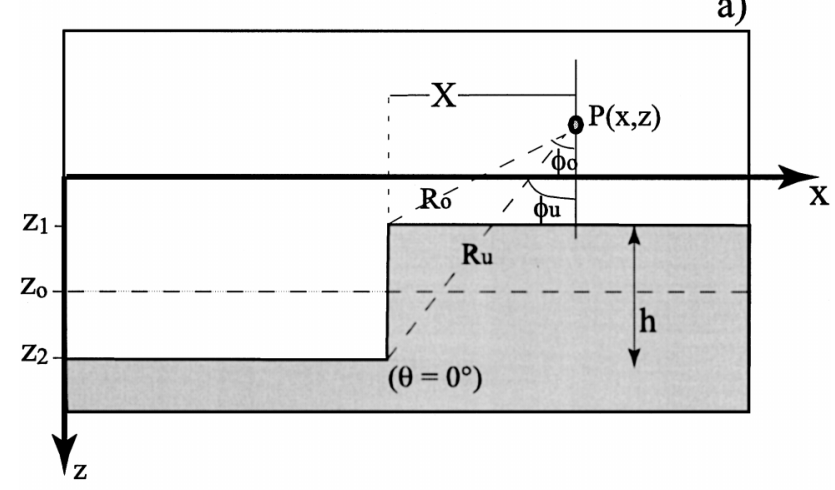

b)

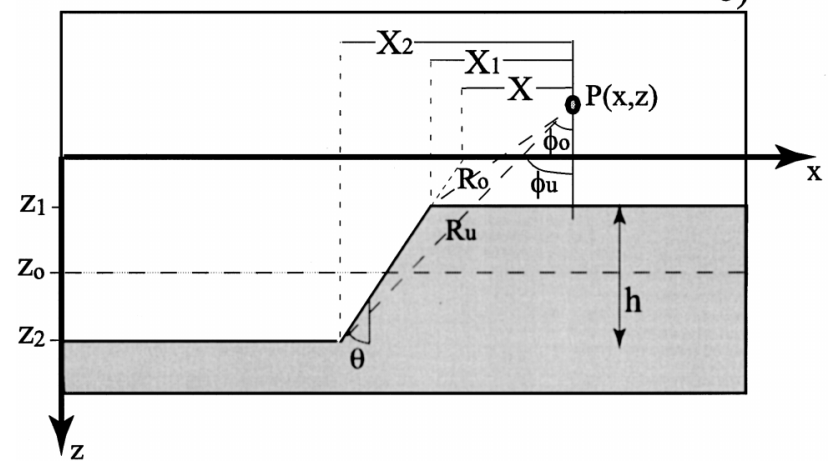

FIG. 2. Cross-sectional view of (a) the vertical step model and (b) the inclined model, showing the notations used throughout this paper. The thin-dyke model corresponds to the inclined edge of the step. 
The first-order term corresponds to a local source located at depth $z_{0}$, with scaling exponent $\beta=-1$ (that is, $\alpha=-1$ and $N=0)$. Higher order terms describe the perturbation induced by the vertical extension of the step.

At this point, we discuss the role of this perturbation and the estimation of both the depth $z_{0}$ and the scaling exponent $\beta$. When the source is pointlike, the scaling relation between $\log \left(|\mathcal{W}| / a^{\gamma}\right)$ and $\log (a+z)$ is strictly linear when $z=z_{0}$ (see Figure 1c). Using this approach, Moreau et al. (1999) have introduced the first wavelet-based automatic interpretation procedure. Adjusting, in a least-squares sense, a straight line to the $\log -\log$ plot for a set of a priori $z$ provides both the depth $z_{0}$ and the scaling exponent $\beta$ of the source. When the source is no longer pointlike, even when $z=z_{0}$, the previous log-log plot exhibits a curved shape with a linear tendency at high dilations only (see Figure 3a). The curved part of the log-log plot (corresponding to low dilations) strongly influences the L2 misfit and biases the estimates of $z_{0}$ and $\beta$. The more extended the source, the stronger this effect. Following this observation, we propose a modification to the original technique: the minimization is now performed using a trial-and-error procedure over the dilation ranges. Starting with the entire range of dilations, we successively test the L2 misfit between the log-log plot and its linear fit over a series of decreasing dilation ranges. We select the dilation range for which the variance of the misfit is minimal. Corresponding slope $\beta$ and depth $z_{0}$ characterize the studied source. Results obtained for the vertical 2-D step using both the method of Moreau et al. (1999) and our technique are shown in Figure 4a. As expected, for strongly extended sources $\left(h / z_{0} \rightarrow 2\right)$, the improvement of the technique we propose is quite effective, with resulting errors $<25 \%$.

We now go further and provide an iterative scheme in which we simultaneously invert for $z_{0}, \beta$, and an intensity parameter $K$ (which depends on both the density contrast and the
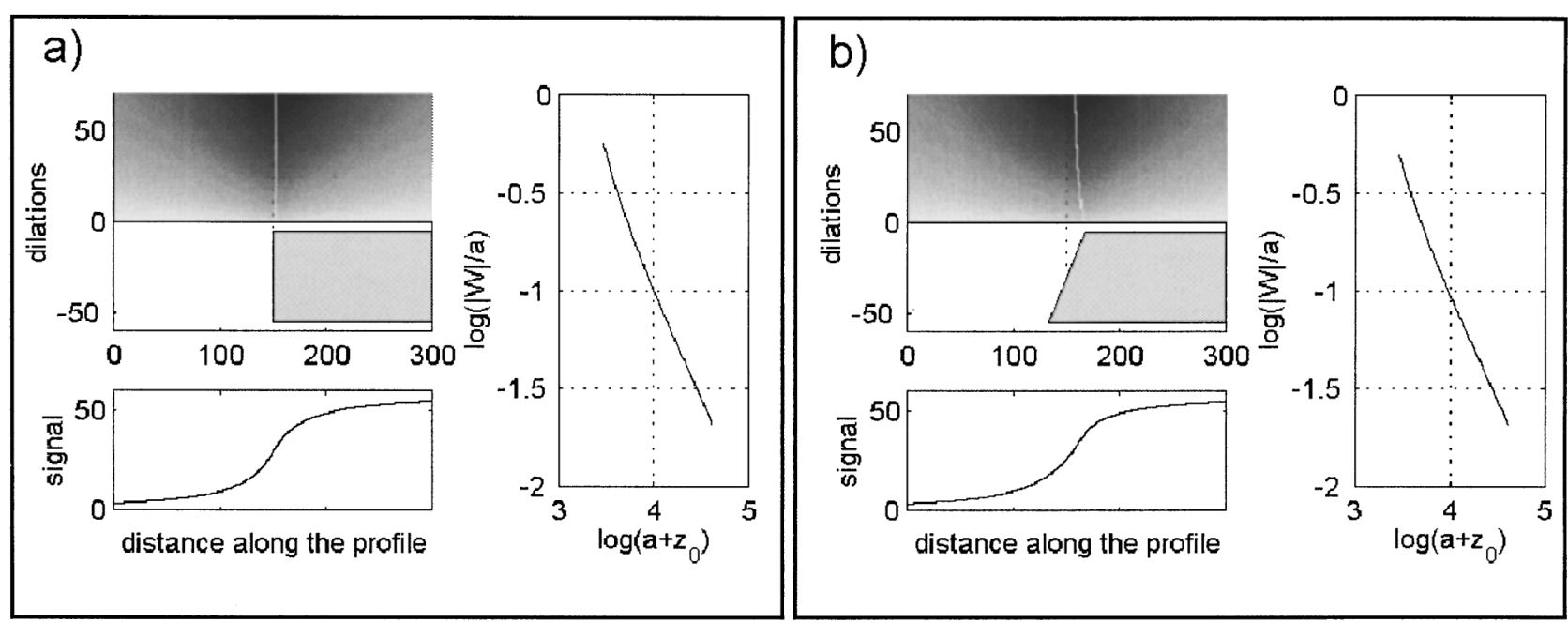

c)
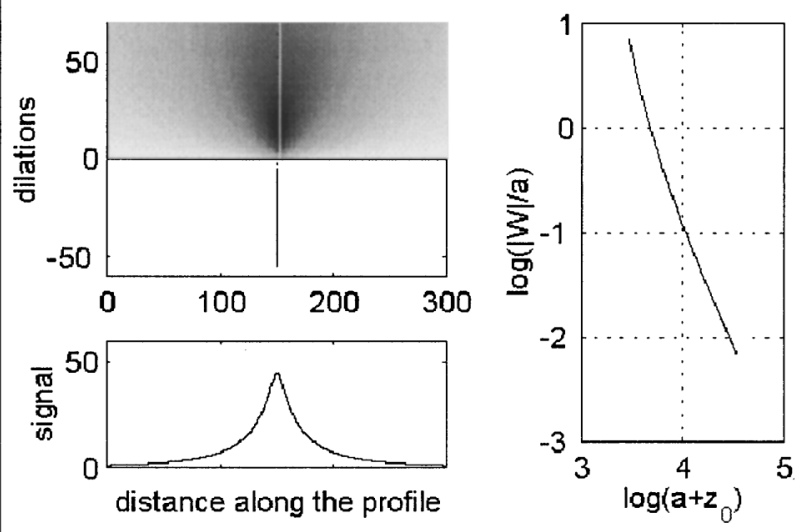

d)
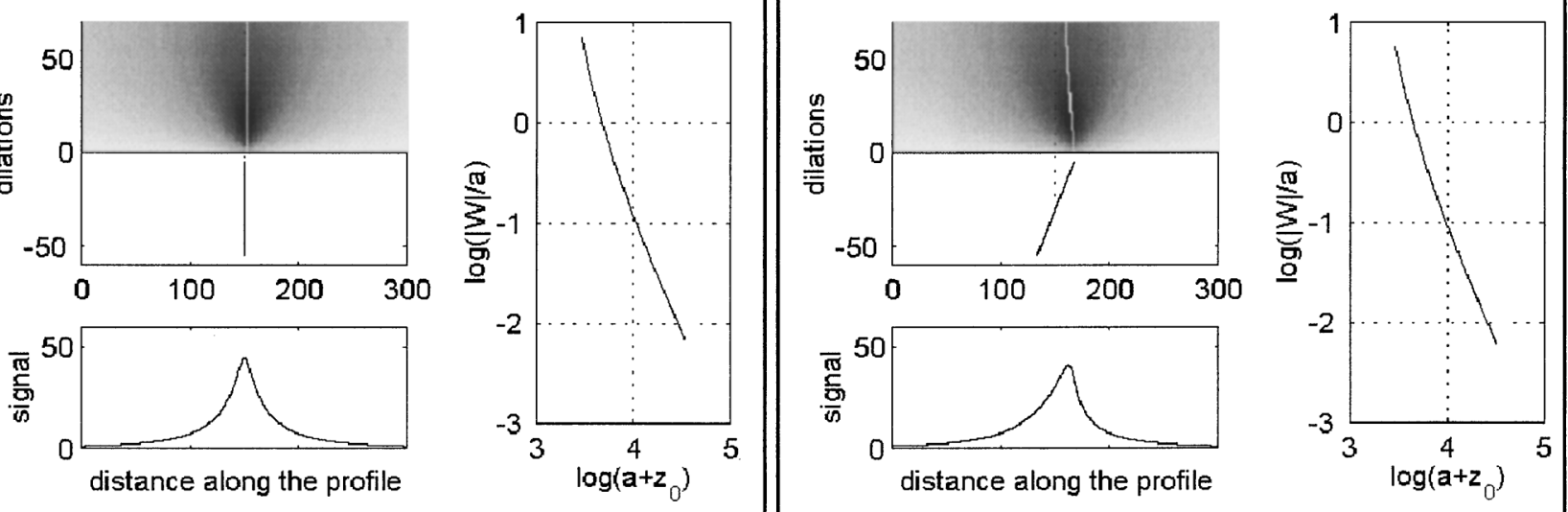

FIG. 3. First-order complex-wavelet transform coefficients of the gravity field. In each case, the associated gravity field and scaling relation between $\log \left(\left|\mathcal{W}_{a}\right| / a\right)$ and $\log \left(a+z_{0}\right)$ are also show. (a) Vertical step, (b) inclined step, (c) vertical dyke, and (d) inclined thin dyke. For vertical sources ( $a$ and c), the modulus maxima line remains straight across the scales. For inclined sources (b and d) at large dilations, this line asymptotically points toward the barycenter of the source. For low dilations, it is curved toward the upper edge of the source. Since all these sources are extended, their scaling relation do not exhibit a perfect linear trend over the entire range of dilations. High dilations are to be preferentially considered to determine the scaling exponent $\beta$ since they represent the bulk source. 
inclination of the step) to recover the thickness $h$ of the step source. Following Sailhac et al. (2000), equation (21) can be rewritten in the form (Appendix B)

$$
\begin{aligned}
\ln & \frac{\left|W_{\psi_{c}^{1} \mid \delta g_{z}(., z=0)}(X=0, a)\right|}{a} \\
& =K+\beta \ln \left(z_{0}+a\right)+R\left(a ; z_{0}, \beta, K\right),
\end{aligned}
$$

where $R$ represents the perturbation induced by the vertical extension of the step. In Appendix B, $R$ is used to derive a function $H\left(a ; z_{0}, \beta, K\right)$, which is an estimator of the thickness $h$ of the step. The set of parameters $\left(z_{0}, \beta, K\right)$ that minimizes the fluctuations of $H\left(a ; z_{0}, \beta, K\right)$ provides the thickness $h$ of the step. Practically, to limit the domain of investigation during the iterative process, initial values of the three parameters are the results of the previous trial-and-error stage. Results obtained for the average depth $z_{0}$ of the vertical 2-D step are plotted in Figure $4 \mathrm{a}$, together with those obtained using the two previous techniques. For strongly extended sources $\left(h / z_{0} \rightarrow 2\right)$, the improvement is very effective (but is still not perfect since the Taylor expansion implies that we are neglecting a term proportional to $h /\left(z_{0}+a\right)$ to the power of five). The accuracy of the estimation of the average depth $z_{0}$ of the step is better than $85 \%$. When $h / z_{0} \rightarrow 0$, the assumption of
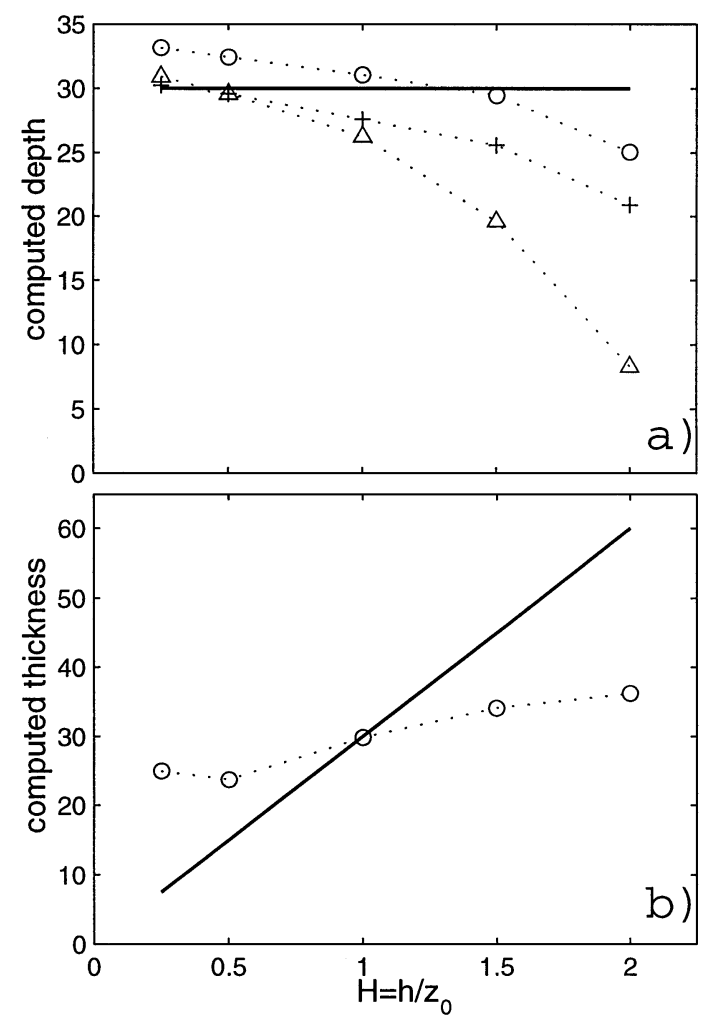

FIG. 4. Plot of the computed average depth and thickness in the case of the vertical step. The influence of the ratio $H$ between the true thickness $h$ and the true average depth $z_{0}$ of the step is emphasized. (a) Computed average depth. Triangles correspond to results obtained using the technique proposed by Moreau et al. (1999). Crosses are obtained by the trial-and-error technique we propose; circles are computed using the inversion scheme. (b) Computed thickness of the step. In (a) and (b), the plane line represents the true average depth and thickness, respectively. extended source is not well suited; therefore, results obtained by the trial-and-error technique (or even the original method by Moreau et al., 1999) are preferred. In addition, Figure 4b presents the estimation of the thickness $h$ of the vertical step. Whether or not perfect, these results can be considered reasonable first-order estimates of the thickness $h$ of the source. When the assumption of extended source holds, the discrepancy between computed and true thicknesses does not exceed 30\%; otherwise, the ratio of the true to the computed thicknesses reaches three.

\section{The inclined step}

We now consider an outcropping inclined 2-D step with its edge having an inclination $\theta$ with respect to the vertical (Figure $2 \mathrm{~b})$. The step of thickness $h$ extends between $z_{1}=z_{0}-(h / 2)$ and $z_{2}=z_{0}+(h / 2)$, with average depth $z_{0}$ and a positive density contrast. With this geometry, expression of the wavelet coefficients equivalent to equation (17) is (Appendix A)

$$
\begin{aligned}
& \mathcal{W}_{\psi_{c}^{1} \mid g_{z}(., z=0)}(x, a) \\
& =G \rho a\left\{\left(\phi_{u}-\phi_{0}\right) \sin 2 \theta+\cos ^{2} \theta \ln \left(\frac{R_{u}^{2}}{R_{0}^{2}}\right)\right. \\
& \left.\quad+i\left[2\left(\phi_{0}-\phi_{u}\right) \cos ^{2} \theta+\cos \theta \sin \theta \ln \left(\frac{R_{u}^{2}}{R_{0}^{2}}\right)\right]\right\},
\end{aligned}
$$

with modulus

$$
\begin{aligned}
& \left|\mathcal{W}_{\psi_{c}^{1} \mid g_{z}(., z=0)}(x, a)\right| \\
& =G \rho a\left\{\left[\left(\phi_{u}-\phi_{0}\right) \sin 2 \theta+\cos ^{2} \theta \ln \left(\frac{R_{u}^{2}}{R_{0}^{2}}\right)\right]^{2}\right. \\
& \left.\quad+\left[2\left(\phi_{0}-\phi_{u}\right) \cos ^{2} \theta+\cos \theta \sin \theta \ln \left(\frac{R_{u}^{2}}{R_{0}^{2}}\right)\right]^{2}\right\}^{1 / 2}
\end{aligned}
$$

and phase

$$
\begin{aligned}
& \Phi_{\psi_{c}^{1} \mid g_{z}(., z=0)}(x, a)=\tan ^{-1} \\
& \left(\frac{2\left(\phi_{0}-\phi_{u}\right) \cos ^{2} \theta+\cos \theta \sin \theta \ln \left(\frac{R_{u}^{2}}{R_{0}^{2}}\right)}{\left(\phi_{u}-\phi_{0}\right) \sin 2 \theta+\cos ^{2} \theta \ln \left(\frac{R_{u}^{2}}{R_{0}^{2}}\right)}\right) .
\end{aligned}
$$

Using the same approach as for the vertical step, we derive both the average depth and thickness of the inclined step for the case $\theta=30^{\circ}$ (see Figure 5). The results and accuracies we obtain are very similar to those derived for the vertical step case: both the trial-and-error and the inversion schemes improve the determination of the average depth $z_{0}$ of the step, especially in the case of extended sources $\left(h / z_{0} \rightarrow 2\right)$. Estimates of the thickness $h$ of the step are also comparable to those obtained for the vertical step. When the step outcrops, 
Figure 6 shows the influence of the inclination of the step on the determination of (a) its average depth and (b) its thickness. Estimates of depth made by the method of Moreau et al. (1999) remain stable independently of the inclination but provide results $50 \%$ underestimated. In comparison, both the trial-anderror and the inversion scheme we propose provide accurate results for $0 \leq \theta \leq 50^{\circ}$ but become unstable for higher values of the inclination. This is because of the increasing curvature of the modulus maxima line when $\theta$ increases and the subsequent difficulty of the curve-fitting process. Concerning the estimate of the thickness $h$, the accuracy of the results remains within $65 \%$ of the true thickness, independent of the inclination $\theta$ of the step.

As shown on Figure $3 b$ in the case of the inclined step, contrary to the vertical step, the modulus maxima line is no longer straight. For high dilations $\left(z_{0}+a \gg h / 2\right)$, the maxima line is a branch of hyperbola and its asymptote (dotted line in Figure $3 \mathrm{~b}$ ) points toward the barycenter of the step. For low dilations $\left(z_{0}+a \ll h / 2\right)$, the line is curved toward the upper edge of the step (see Figure 3b). Sailhac et al. (2000) show that for the magnetic field, the tangent at low dilations is related to the dipping angle $\theta$ of the step. This is also true for gravity. But here, we do not use this property to recover the dipping angle of the step. Instead, we study the value of the phase at the base of the modulus maxima line, that is, for $a \rightarrow 0^{+}$. This is equivalent to picking the phase at the maximum of the analytic signal- in
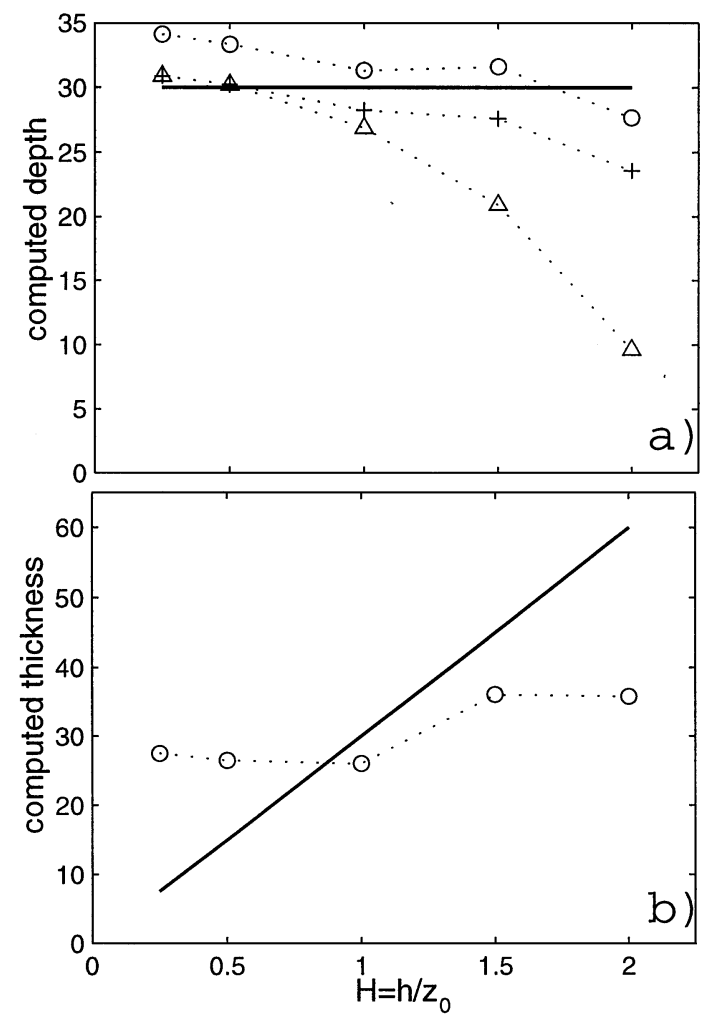

FIG. 5. Plot of the computed average depth and thickness in the case of the inclined step. Symbols are the same as in Figure 4. (a) Computed average depth. (b) Computed thickness of the step. our case, where the edge of the step outcrops $\left(X_{1}=0\right)$. At this point, the phase is given by

$$
\lim _{a \rightarrow o^{+}} \Phi_{\psi_{c}^{1} \mid g_{z}(., z=0)}\left(X_{1}=0, a\right)=\theta .
$$

Figure 7 shows the results obtained numerically for the outcropping inclined step we previously defined. Dipping angle $\theta$ varies from $0^{\circ}$ to $80^{\circ}$ with respect to the vertical. Values of the phase reported in Figure 7 are picked out for dilations of 0 (equivalent to the analytic signal), 2 , and 4 . Using this technique, we are able to estimate the dip of the source with an underestimation of about $10-20 \%$. In addition, obtained curves clearly exhibit the influence of the noise at the ground level and also the decrease of the phase toward zero when higher dilations are considered. Indeed, this behavior is well known in the potential field domain: close to the source, the field holds much information but is noisy; far from the source, the field is less irregular but has lost most of the short-wavelength information.

Another way to recover the dipping angle of the source without being as sensitive to the noise at low dilations is derived from equation (25). We investigate the locations where
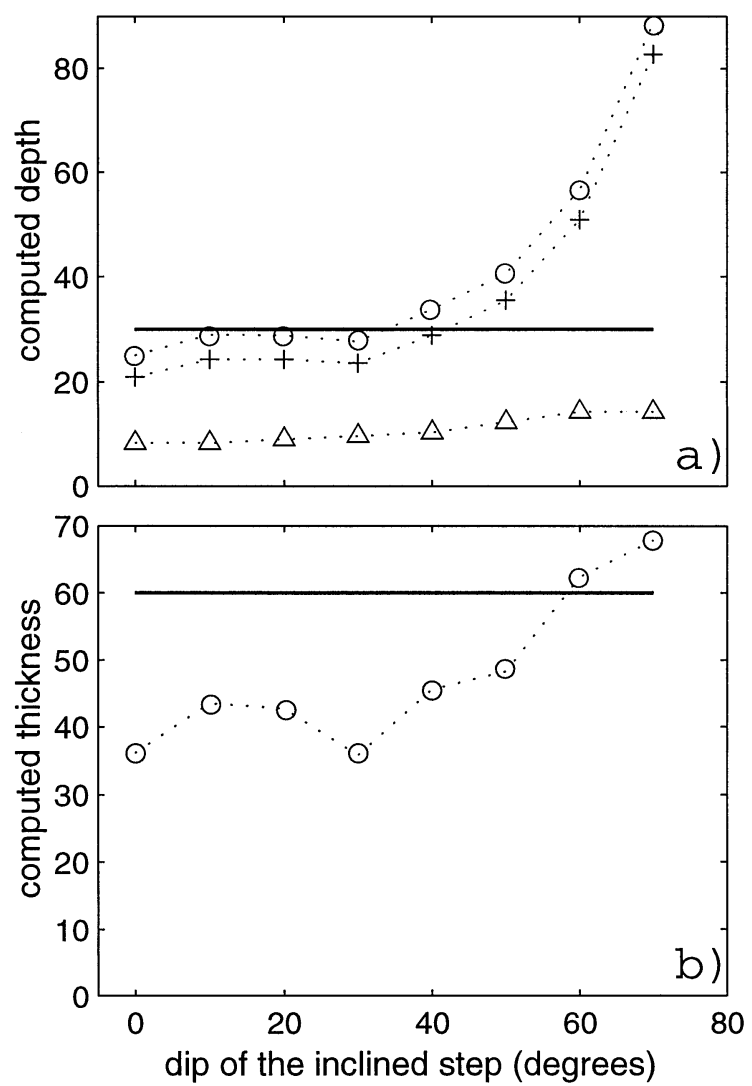

FIG. 6. Plot of the computed average depth and thickness in the case of the inclined step. The influence of the dip $\theta$ of the step is emphasized. (a) Computed average depth. Triangles correspond to results obtained using the technique proposed by Moreau et al. (1999). Crosses are obtained by the trial-and-error technique, and circles are computed using the inversion scheme. (b) Computed thickness of the step. In (a) and (b), the plane line represents the true average depth and thickness, respectively. 
the phase of the complex wavelet transform is equal to $\theta$ (the dipping angle of the step). Since $\mathcal{W}_{\psi_{c}^{1} \mid g_{z}(., z)}=a\left(\partial_{x} g+\right.$ $\left.i \partial_{z} g\right)(x, z+a)$, such points are given by $\partial_{x} g \tan \theta=\partial_{z} g$, that is,

$$
\begin{gathered}
2\left(\phi_{u}-\phi_{0}\right) \tan ^{2} \theta+\tan \theta \ln \left(\frac{R_{u}^{2}}{R_{0}^{2}}\right) \\
=2\left(\phi_{0}-\phi_{u}\right)+\tan \theta \ln \left(\frac{R_{u}^{2}}{R_{0}^{2}}\right) .
\end{gathered}
$$

Therefore, we see that for $\phi_{u}=\phi_{0}$, i.e., in the alignment of the edge of the step, the phase is constant and equal to $\theta$. This particular property is suggested by Sailhac et al. (2000) and, for the field itself, by Paul (1966). In fact, in the case of a local homogeneous source, the isophase lines are straight and define a conelike structure pointing toward the source. For extended inclined sources, isophase lines are no longer straight. At low dilations they are curved and converge toward the upper part of the source (see Figure 8), except for the line corresponding to $\phi_{u}=\phi_{0}$, which remains straight. This approach is convenient for determining the dipping angle of the source since, contrary to the exposed technique, it is not strongly influenced by data noise. Still, the method can be applied as long as the source is not too deep. Otherwise, it can be considered a local source and every isophase line becomes straight.

\section{Inclined and vertical dykes}

The last synthetic case we consider is a 2-D thin dyke. Here we expose the general case of the inclined dyke with an angle $\theta$. (A vertical dyke corresponds to $\theta=0$.) The dyke extends between $z_{1}=z_{0}-(h / 2)$ and $z_{2}=z_{0}+(h / 2)$ with average depth $z_{0}$ and a positive density contrast.

Using Poisson's relation, we can convert the total magnetic field into the pseudogravity field (Baranov, 1957). Here we use this property to derive the analytic expressions corresponding to our gravity dyke. In the wavelet domain, $(\gamma+1)-$ order wavelet coefficients of $g_{z}$ (the vertical gravity field) are asso-

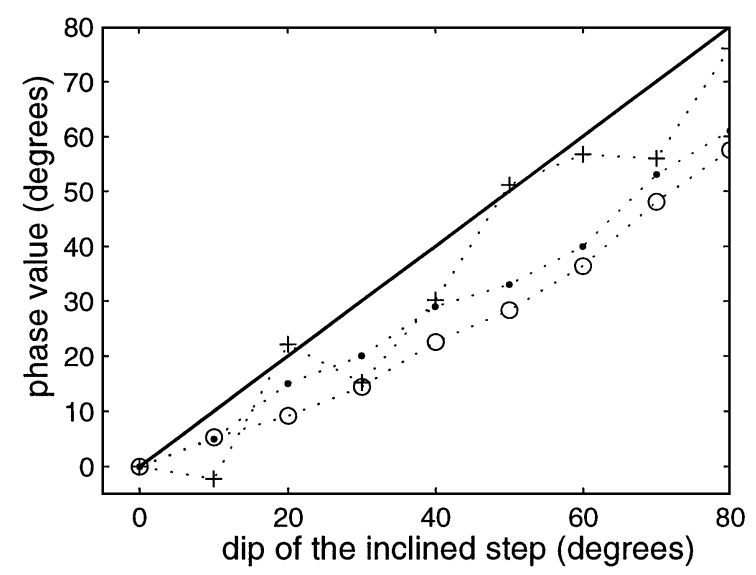

FIG. 7. Case of the $\gamma=1$ complex wavelet of the outcropping inclined step. Plot of the value of the phase at the base of the modulus maxima line versus the dip of the step. According to equation (26), both terms should be equal, which is represented by the straight plane line. Crosses correspond to values of the phase picked at the dilation 0 , dots at dilation 2 , and circles at dilation 4. ciated to $\gamma$-order wavelet coefficients of $T$ (the total magnetic field) via (Sailhac et al., 2000)

$$
\mathcal{W}_{\psi_{c}^{\gamma+1} \mid g_{z}(., z)}(x, a)=a \mathcal{W}_{\psi_{c}^{\gamma} \mid T(., z)}(x, a)
$$

in the case of a normal and vertical magnetic field. Thus, we can derive the analytical expression of the $\gamma=1$ wavelet transform of the gravity field of the dykelike source from the one of the total magnetic field of a steplike source:

$$
\begin{aligned}
& \mathcal{W}_{\psi_{c}^{1} \mid g_{z}(., z=0)}(x, a) \\
& \quad=2 G \rho a h\left[\frac{1}{\left(z_{2}+a\right)+i X_{2}}-\frac{1}{\left(z_{1}+a\right)+i X_{1}}\right] .
\end{aligned}
$$

The associated modulus is

$$
\begin{aligned}
& \left|\mathcal{W}_{\psi_{c}^{1} \mid g_{z}(., z=0)}(x, a)\right| \\
& =\frac{2 G \rho a h}{\sqrt{\left[X_{1}^{2}+\left(z_{1}+a\right)^{2}\right]\left[X_{2}^{2}+\left(z_{2}+a\right)^{2}\right]}}
\end{aligned}
$$

and phase is

$$
\begin{aligned}
& \Phi_{\psi_{c}^{1} \mid g_{z}(., z=0)}(x, a) \\
& \quad=-\left[\tan ^{-1}\left(\frac{z_{1}+a}{X_{1}}\right)+\tan ^{-1}\left(\frac{z_{2}+a}{X_{2}}\right)\right] .
\end{aligned}
$$

The correspondence between the total magnetic field of a steplike source and the vertical component of the gravity field of a dykelike source has been shown. Since Saillhac et al. (2000) extensively develop the analytic expressions for deriving $z_{0}, \beta$, and $h$ of the magnetic step, we will only show the numerical results obtained on the corresponding gravity dyke. Using the same approach as described in the step, we derive in Figure 9 both the average depth $z_{0}$ and the vertical extension $h$ of the dyke for (a) the vertical dyke and (b) the inclined one. Globally, accuracy of the estimation of both $z_{0}$ and $h$ for the dyke are comparable to that obtained for the step.

\section{FIELD EXAMPLE}

The wavelet transform method is used on gravity data we measured in central Nepal during an IDYLHIM FrenchNepalese cooperation project. We collected 80 gravity measurements along a north-northeast-south-southwest profile, perpendicular to the Himalayan range (Figure 10). The profile crosses the main thrusting structures outcropping at the front of the range as the result of the India-Asia collision. These thrusts are generally interpreted in an evolutionary model (e.g., Le Fort, 1975). The shortening between India and Asia is successively accommodated by several decollement structures migrating southward (see Figure 10), namely, the main central thrust (MCT), the main boundary thrust (MBT), and the presently most active main frontal thrust (MFT). At depth, all these thrusts are underlain and linked via the flat-ramp-flat main horizontal thrust (MHT), which represents the upper limit of the underthrusting Indian plate. The measured gravity profile is $150 \mathrm{~km}$ long, sampled every 1-4 km. Classical corrections have been applied to the data set, including terrain corrections computed up to $167 \mathrm{~km}$ (Martelet et al., 1997). Prior to wavelet processing, the resulting complete Bouguer anomaly profile was interpolated every $1 \mathrm{~km}$ using a 
cubic spline (Figure 11a). It was extended to the north and south using data from China (Sun, 1989) and India (extracted from the Bureau Gravimétrique International database) to avoid edge effects.

Wavelet transform coefficients were computed along the profile using $\partial^{2} / \partial x^{2}, \partial^{3} / \partial z \partial x^{2}$, and $\partial^{3} / \partial x^{3}$ derivatives. In Figure 11b, we show the wavelet coefficients (grayshades) and associated modulus maxima lines (dots) obtained in the case of the third horizontal derivative $\left(\partial^{3} / \partial x^{3}\right)$. When possible, we superimposed continuations of the maxima lines in the lower half-plane (plane lines). Their intersections allow us to locate several sources. In Figure 12, we summarize all the localizations obtained geometrically using the three derivatives of the real wavelet. When sources are extended, the obtained localizations range between the top and the middle of the source, depending on the order of derivation of the wavelet. Generally, the higher the order of derivation, the shallower the intersection of the maxima lines. Sources shown in Figure 12 are all located between the surface and the MHT decollement. This result is satisfactory since short-wavelength gravity anomalies along the profile are generally interpreted by lateral contrasts of density that occur above the MHT. Figure 13 summarizes all the localizations, shape indices, thicknesses, and dips of the sources obtained using first and second derivatives of the complex wavelet. For these determinations we used the methods of computation that we exposed before, namely, the simultaneous inversion of average depth, homogeneity degree and thickness of the source, and picking of the phase at the base of the complex modulus maxima lines. Again, sources appear to be located between the surface and the MHT decollement. In addition, we get information concerning the homogeneity degree of the source (i.e., its shape), its inclination, and vertical extension. The thicknesses we determine here are not taken into account for further interpretations since all the studied contacts outcrop. Nevertheless, the computed vertical extensions of the sources appear reasonable. Using the results of Figures 12 and 13, we infer, in terms of simple-shaped contacts, the interpretive section of Figure 14. Results are superimposed on a structural cross-section (J. Ph. Avouac, personal communication, 1998) derived from surface geology and seismologic active and passive experiments (e.g., Schelling and Arita, 1991; Zhao et al., 1993; Pandey et al., 1995).

It turns out that beneath the Lesser Himalaya, step-shaped sources (black dots in Figure 14), reach depths of 6 to $8 \mathrm{~km}$, in good agreement with the previously estimated depth of the flat, subhorizontal detachment. The sources dip northward at angles of about $30^{\circ}$. All these estimate are comparable to what was deduced from surface geological observations and focal mechanism relocalizations. Farther north, the smoother gravity variations beneath the Higher Himalaya are related to deeper sources. Their location are consistent with the position of deeper thrusts that merge with the midcrustal detachment surface at about $20 \mathrm{~km}$ depth. For shallow depths their signature is comparable to that of a dyke. This can be explained
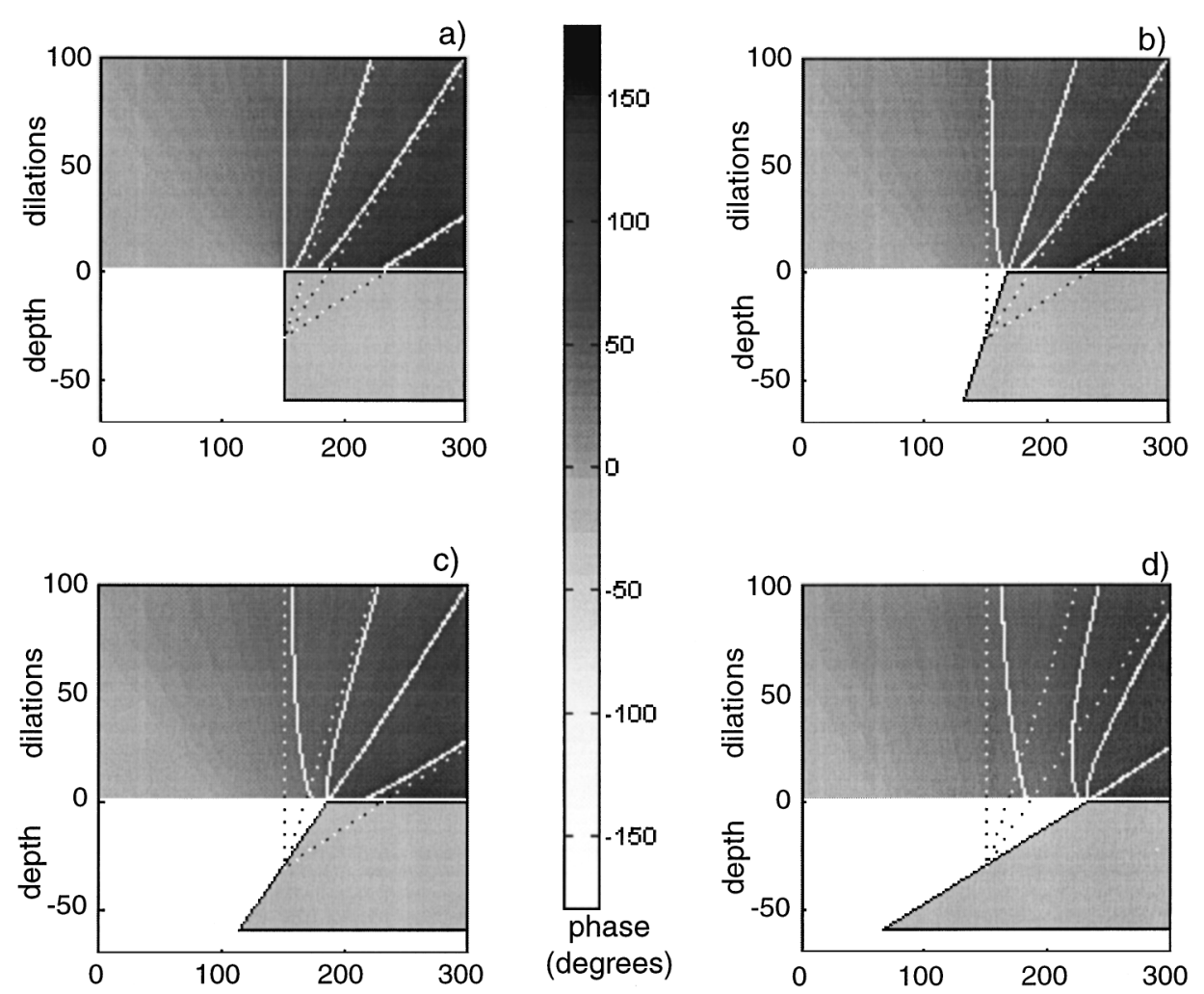

FIG. 8. Plot of the phase of the $\gamma=1$ complex wavelet of the outcropping inclined step across the scales. Dip $\theta$ of the steps is (a) $0^{\circ}$, (b) $30^{\circ}$, (c) $50^{\circ}$, and (d) $70^{\circ}$. Superimposed $0^{\circ}, 30^{\circ}, 50^{\circ}$, and $70^{\circ}$ equiphase lines have the same value as the dip of the step in the alignment of the edge of the step. Moreover, they remain straight across the scales only in the alignment of the edge of the step. Once the step source has been localized, this is a convenient way to determine its dip. 
by the fact that these structures are ancient major thrusts in which the rocks have been strongly crushed and therefore form a thick gouge. Deeper, the signature evolves toward a step. Also, in Kathmandu klippe, our method detects the presence of a granite massif (see Figure 10). Its vertical extension is about 7 to $8 \mathrm{~km}$. This suggests the granites do not cross the flat detachment. On the contrary, they appear to lie on this tectonic contact. Moreover, our measurement sampling is thin enough to study both the limits of the massif using low dilations or to study the massif on the whole when considering high dilations. This behavior is clearly visible in Figure $11 \mathrm{~b}$ at $x \sim 50 \mathrm{~km}$ along the profile. At high dilations, only one maxima line exists (it characterizes the whole massif); for dilations lower than $10 \mathrm{~km}$, this line splits into two lines that point toward the southern and northern limits of the granite.

\section{CONCLUSION}

Gravity interpretation suffers from the well-known nonuniqueness of the inversion. Consequently, it demands additional a priori information. Several existing methods let us analyze the gravity field prior to modeling to extract this information. The upward continuation operator is often used to enhance the characteristics of a measured potential field at several wavelengths. Also, it has long been recognized that combining the derivatives of the gravity field yields useful informations concerning the causative sources. Indeed, assuming simple-shaped sources, their depths can be recovered (e.g., Euler deconvolution or analytic signal methods).

The wavelet method we present combines the upward continuation and derivation operators into a new, convenient for- malism. First, it can be used in a geometric manner to recover simultaneously the average depth and shape of causative sources (assuming the sources are homogeneous). This geometric technique is applied on any derivatives of the real wavelet modulus, depending on which specific property of the source is emphasized. Lines of the maxima of the modulus drawn across the dilation scales point to the source. Along these maxima lines, the wavelet modulus exhibits a double scaling law. For a pointlike source, the slope coefficients so determined provide the homogeneity degree of the source, i.e. its shape. In the case of extended sources, a second-order term appears in the scaling law. A simple L2 misfit computation over the entire range of dilations is no longer accurate. Therefore, we propose a trial-and-error approach that focuses on particular ranges of dilations. This technique improves the joint determination of shape and average depth of the source, especially when the vertical extension of the source is significant. Complex wavelet transform can also be computed as the sum of $\psi_{x}^{\gamma}$ for the real part and its Hilbert transform $\mathcal{H}\left[\psi_{x}^{\gamma}\right]=-\psi_{z}^{\gamma}$ for the imaginary part. This operator modulus corresponds to the analytic signal that would have been computed at all levels above the level of measurements. For this operator we derive analytically an estimator of the thickness $h$ of the extended source. Taking the output of the previous trial-and-error stage as a starting point, we iteratively derive $h$ and, simultaneously, the average depth $z_{0}$ and shape factor $\beta$ in the case of vertical or inclined step and dyke sources. Tests performed on synthetic sources show a very good accuracy of the determined depths and a good first approximation of the computed thicknesses. Finally, analytic results also allow determination of the dipping angle of the a)
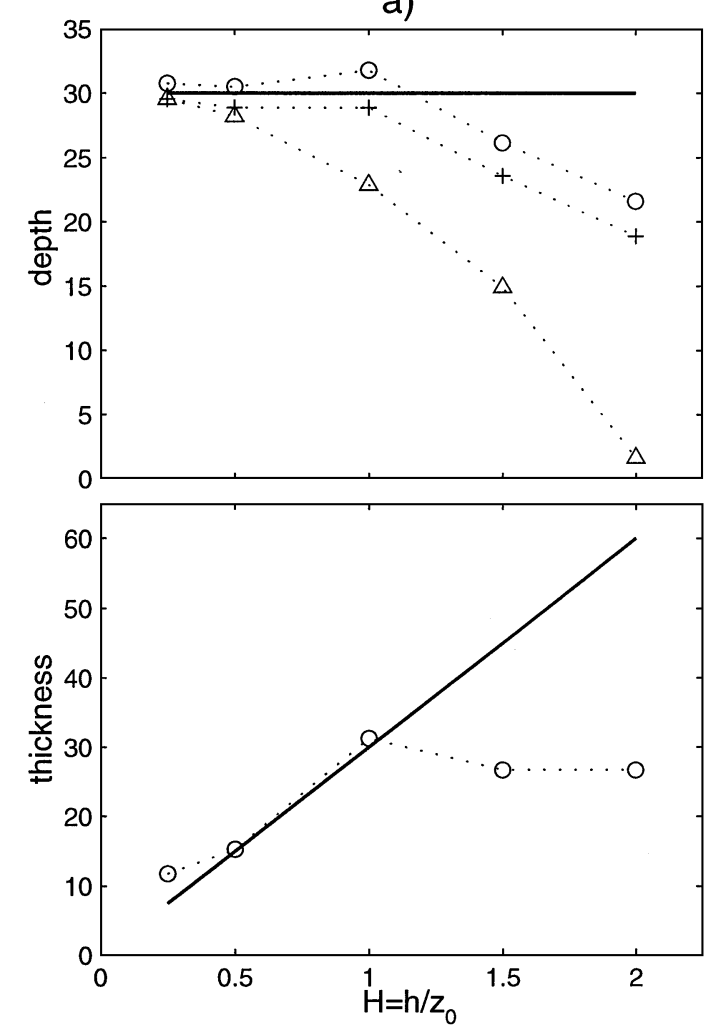

b)
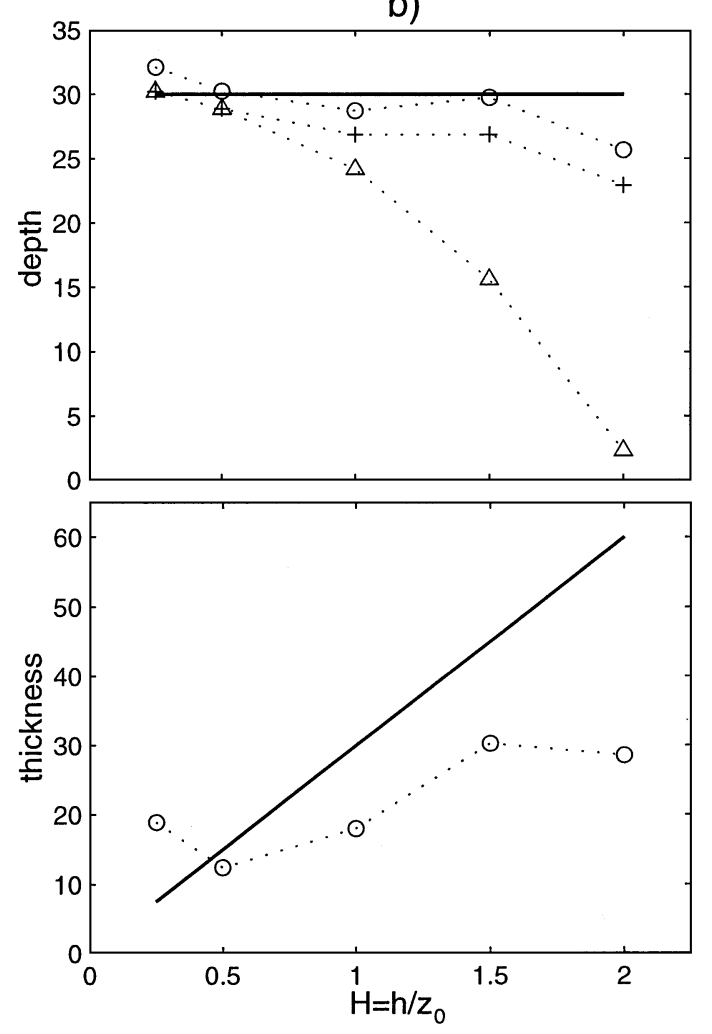

FIG. 9. Plot of the computed average depth and thickness in the case of the dyke. (a) For a vertical dyke. (b) For an inclined dyke. Symbols are the same as in Figure 4. 


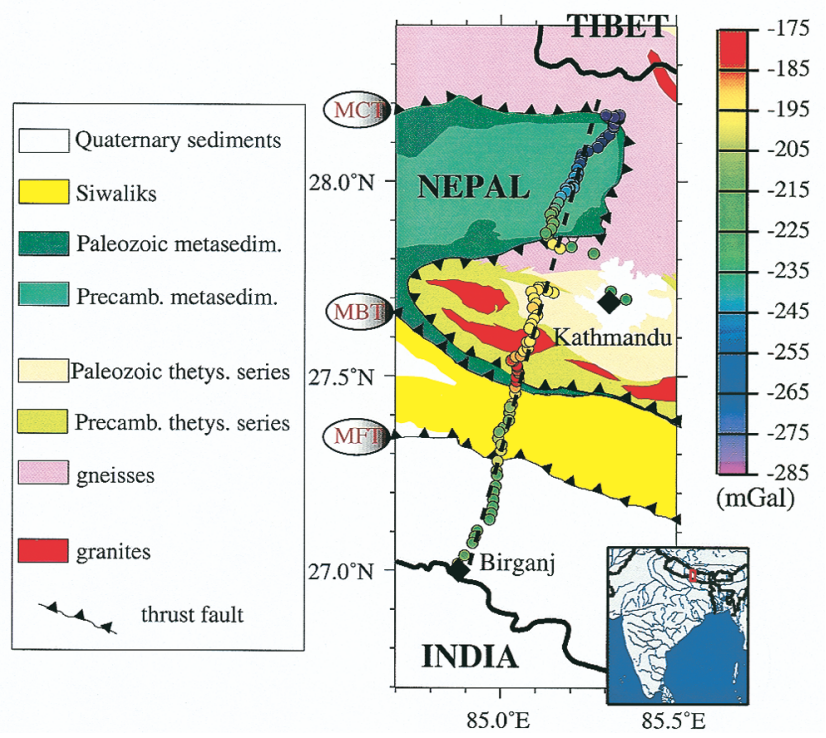

FIG. 10. Simplified geological map with location of the gravity profile measured across the Himalayas of Nepal. Colors of the circles show the complete Bouguer anomaly. Our data perpendicularly cross several thrusting structures outcropping at the front of the collision range. Also, in Kathmandu klippe they cross a zone of granitic massifs. source from the phase term of the complex wavelet transform. Synthetic tests show that the computed inclination of the source is generally good even if slightly underestimated. Application of these techniques to a gravity profile we measured across the Himalayas of Nepal (1) provides results in good agreement with previously well-recognized structures and therefore

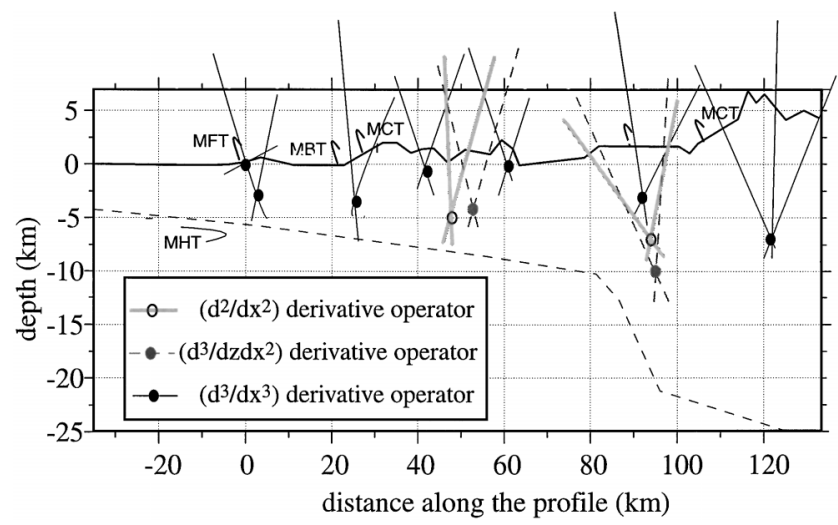

FIG. 12. Recapitulation of all the localizations of sources geometrically obtained along the Nepalese profile. Three different derivation operators of the real wavelet have been used. The black dashed line corresponds to the MHT flat-ramp-flat deep detachment (as inferred by active and passive seismological experiments).

a)
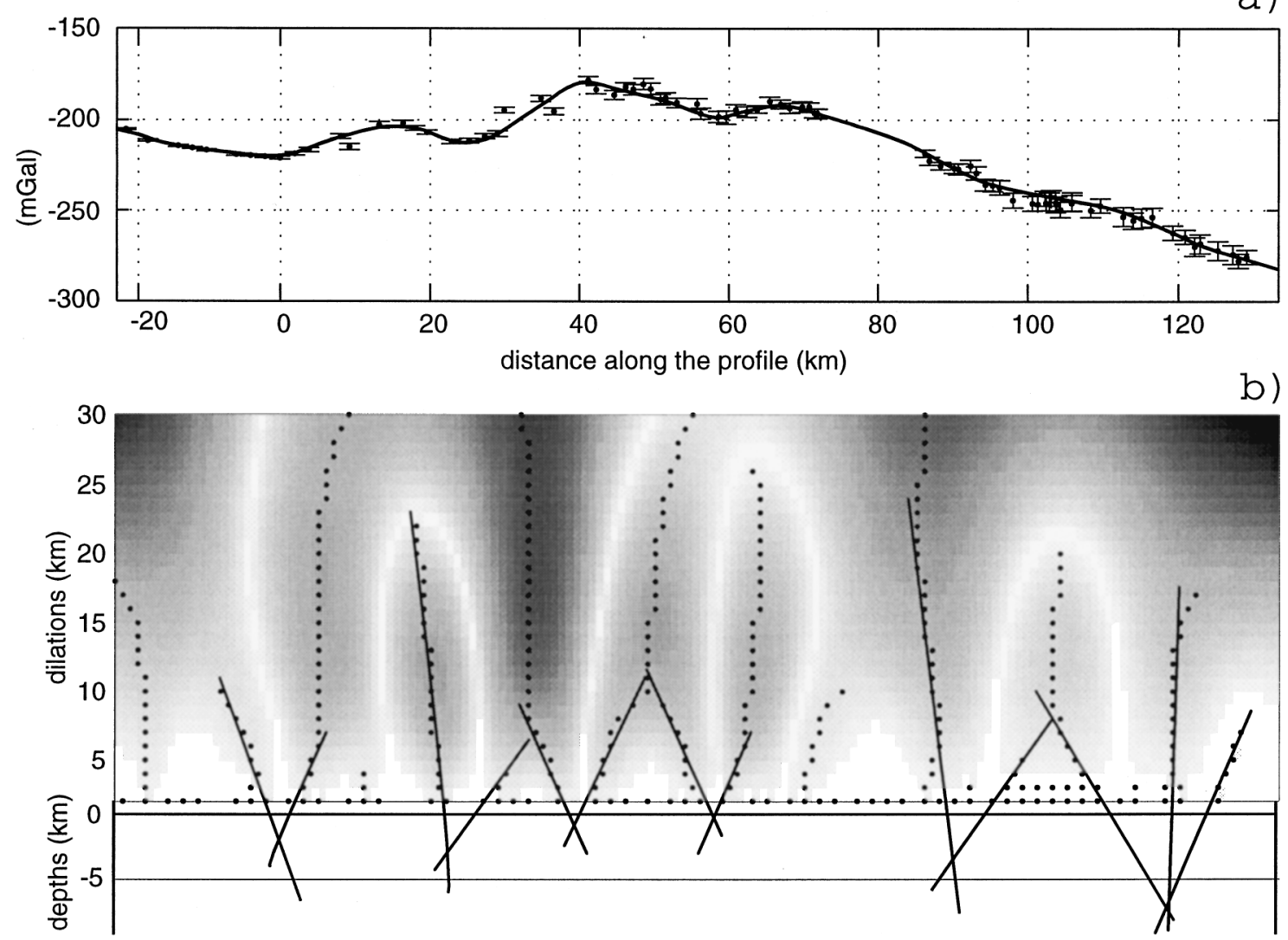

FIG. 11. (a) Complete Bouguer anomaly along our profile. Both the data (with error bars) and the 1-km interpolated anomaly are presented. (b) Wavelet coefficients (third-order horizontal derivative) across the scales. Black dots represent the maxima of the modulus. Superimposed black lines locate sources in the lower half-plane. 


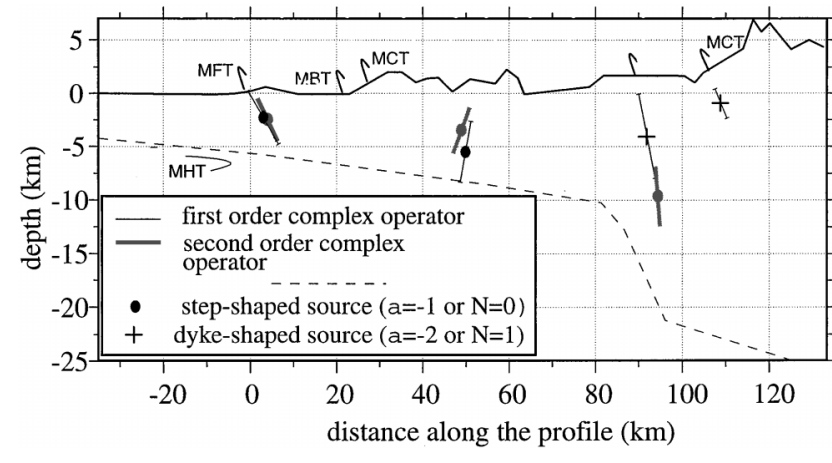

FIG. 13. Recapitulation of all the localizations, shape indices, thickness, and dip of the sources obtained using $\gamma=1$ and $\gamma=2$ complex wavelets. Simultaneous inversion of average depth, homogeneity degree, and thickness of the source was performed using equation (B-9). The dip was obtained by picking the value of the phase at the base of the modulus maxima lines.

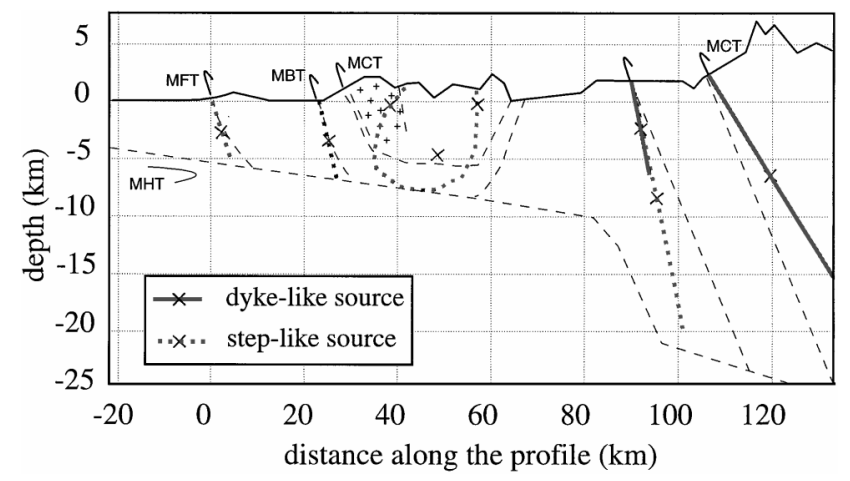

FIG. 14. Interpretive cross-section, taking into account results obtained both in a geometric way (Figure 12) and by numeric computations (Figure 13). Interpretations are superimposed on a structural cross-section (dashed lines) that summarizes present knowledge of the Himalayan crustal structure (as inferred by seismology). Step-shaped contacts are represented by dots; dykelike ones, by plane lines. We observe generally good agreement between the sources we infer and the stuctures previously recognized. In addition, in the deeper parts of the section where the structures are not well documented, our results bring new constraints.

demonstrates the applicability of the method to real data and (2) allows inference of the approximate geometries of other less constrained deep geological structures.

\section{ACKNOWLEDGMENTS}

We thank Dominique Gibert and Matthias Holschneider for fruitful discussions as well as R. S. Pawlowski and E. K. Beigert for constructive reviews. Data were acquired under the support of the CNRS/INSU-IDYLHIM program. This is IPGP publication no. 1670.

\section{REFERENCES}

Baranov, V., 1957, A new method for interpretation of aeromagnetic maps: Pseudogravimetric anomalies: Geophysics, 22, 359-383.

Barbosa, V. C. F., Silva, J. B. C., and Medeiros W. E., 1999, Stability analysis and improvement of structural index estimation in Euler deconvolution, Geophysics, 64, 48-60.
Bhattacharyya, B. K., 1972, Design of spatial filters and their application to high-resolution aeromagnetic data: Geophysics, 37, 68-91.

Blakely, R. J., 1995, Potential theory in gravity and magnetic applications: Cambridge Univ. Press.

Chastenet, de Gery T., and Naudy, H., 1957, Sur l'interprétation des anomalies gravimétriques et magnétiques Geophys. Prosp., 5, 421448

Cordell, L., and Grauch, V. J. S., 1985, Mapping basement magnetization zones from aeromagnetic data in the San Juan basin, New Mexico, in Hinze, W.J., Ed., The utility of regional gravity and magnetic anomaly maps: Soc. Expl. Geophys., 181-197.

Debeglia, N., and Corpel, C., 1997, Automatic 3-D interpretation of potential field data using analytic signal derivatives: Geophysics, 62 , 87-96.

Fedi, M., and Quarta, T., 1998, Wavelet analysis for the regionalresidual and local separation of potential field anomalies: Geophys. Prosp., 46, 507-525.

Foufoula-Georgiou, E., and Kumar, P., 1994, Wavelets in geophysics: Academic Press Inc.

Grossman, A., and Morlet, J., 1984, Decomposition of Hardy functions into square integrable wavelets of constant shape: J. Math. Ann., 15, 723-736.

Hartman, R. R., Teskey, D. J., and Friedberg, J. L., 1971, A system for rapid digital aeromagnetic interpretation: Geophysics, 36, 891-918.

Holschneider, M., 1995, Wavelets an analysis tool: Oxford Univ. Press, Inc.

Hornby, P., Boschetti, F., and Horovitz, F. G., 1999, Analysis of potential field data in the wavelet domain: Geophys. J. Internat, 137, 175-196.

Hsu, S.-K., Coppens, D., and Shyu, C.-T., 1998, Depth to magnetic source using the generalized analytic signal: Geophysics, 63, 19471957.

Huang, D., 1996, Enhancement of automatic interpretation techniques for recognising potential field sources: $\mathrm{PhD}$ thesis, Univ. of Leeds.

Keating, P. B., 1998, Weighted Euler deconvolution of gravity data: Geophysics, 63, 1595-1603.

Lefort, P., 1975, Himalayas: The collided range. Present knowledge of the continental arc: Am. J. Sci., 275-A, 1-44.

Marson, I., and Klingele, E. E., 1993, Advantages of using the vertical gradient of gravity for 3-D interpretation: Geophysics, 58, 15881595 .

Martelet, G., Diament, M., Shakya, T. R., and Burov, E. B., 1997, A new gravity survey in Nepal: Constraints on the mechanical properties of the lithosphere and on the crustal structure: Presented at the 1997 fall mtg., Am. Geophys. Union,

Moreau, F., 1995, Méthodes de traitement de données géophysiques par transformée en ondelettes: PhD thesis, Univ. de Rennes I.

Moreau, F., Gibert, D., Holschneider, M., and Saracco, G., 1997, Moreau, F., Gibert, D., Holschneider, M., and Saracco, G., 1997,
Wavelet analysis of potential fields: Inverse Problems, 13, 165-178. Wavelet analysis of potential fields: Inverse Problems, 13, 165-178.
1999, Identification of sources of potential fields with the continuous wavelet transform: Basic theory: J. Geophys. Res., 104, 50035013.

Nabighian, N. N. 1972, The analytic signal of two-dimensional magnetic bodies with polygonal cross-section: Geophysics, 37, 507-517. Nelson, J. B., 1988, Comparison of gradient analysis techniques for linear two-dimensional magnetic sources: Geophysics, 53, 10881095.

Pandey, M. R., Tandukar, R. P., Avouac, J. P., Lavé, J., and Massot, J. P., 1995, Interseismic strain accumulation on the Himalayan crustal ramp (Nepal): Geophys. Res. Lett., 22, 751-754.

Paul, M. K., Datta, S., and Banerjee, B., 1996, Direct interpretation of two-dimensional structural faults from gravity data: Geophysics, 31, 940-948.

Pedersen, L. B., and Rasmussen, T. M., 1990, The gradient tensor of potential field anomalies: Some implications on data collection and data processing of maps: Geophysics, 55, 1558-1566.

Peters, L. J., 1949, The direct approach to magnetic interpretation and its practical application: Geophysics, 14, 290-320.

Pilkington, M., 1997, 3-D magnetic imaging using conjugate gradients: Geophysics, 62, 1132-1142.

Reid, A. B., Allsop, J. M., Granser, H., Millett, A. J., and Somerton. I. W., 1990, Magnetic interpretation in three dimensions using Euler deconvolution: Geophysics, 55, 80-91.

Ridsdill-Smith, T. A., and Dentith, M. C., 1999, The wavelet transform in aeromagnetic processing: Geophysics, 64, 1003-1013.

Ridsdill-Smith, T. A., Dentith, M. C., and List, R. D., 1999, Applications of wavelet transforms in aeromagnetic data processing: SAGA 99, Expanded Abstracts, 158-165.

Roest, W. R., Verhoef, J., and Pilkington, M., 1992, Magnetic interpretation using the 3-D analytic signal: Geophysics, 57, 116-125.

Sailhac, P., Galdéano, A., Gibert, D., Moreau, F., and Delor, C., 2000, Identification of sources of potential fields with the continuous wavelet transform: Complex wavelets and application to aeromagnetic profiles in French Guiana: J. Geophys. Res., 105, 19455-19475. 
Schelling, D., and Arita, K., 1991, Thrust tectonics, crustal shortening and the structure of the far-eastern Nepal Himalaya: Tectonics, 10, $851-862$.

Spector, A., and Grant, F. S., 1970, Statistical models for interpreting aeromagnetic data: Geophysics, 35, 293-302.

Stavrev, P. Y., 1997, Euler deconvolution using differential similarity transformations of gravity or magnetic anomalies: Geophys. Prosp., 45, 207-246.

Sun, W., 1989, Bouguer gravity anomaly map of the People's Republic of China: Acad. of Geoexpl., Beijing.
Telford, W. M., Geldart, L. P., and Sheriff, R. E., 1990, Applied geophysics: Cambridge Univ. Press.

Thompson, D. T., 1982, A new technique for making computerassisted depth estimates from magnetic data: Geophysics, 47, 31 37.

Werner, S., 1953, Interpretation of magnetic anomalies at sheet-like bodies: Sveriges Geologiska Undersok, Ser. C, 43, N06.

Zhao, W., Nelson, K. D., and Team, P. I., 1993, Deep seismic reflection evidence for continental underthrusting beneath southern Tibet: Nature, 366, 557-559.

\section{APPENDIX A}

\section{GRAVITY FIELD OF A STEP AND ITS DERIVATIVES}

At an altitude $-z$ above ground level, the gravity field of a finite inclined 2-D step can be expressed (after Telford et al., 1990) as

$$
\begin{aligned}
g(x, z)= & G \rho\left\{\phi_{u}\left[2\left(z_{2}-z\right)+X \sin 2 \theta\right]\right. \\
& -\phi_{0}\left[2\left(z_{1}-z\right)+X \sin 2 \theta\right] \\
& \left.+X \cos ^{2} \theta \ln \left(\frac{R_{u}^{2}}{R_{0}^{2}}\right)\right\} .
\end{aligned}
$$

Refer to notations and geometry in Figure 2. With

$$
\begin{aligned}
& \left\{\begin{array}{l}
R_{0}^{2}=X_{1}^{2}+\left(z_{1}-z\right)^{2}, \\
R_{u}^{2}=X_{2}^{2}+\left(z_{2}-z\right)^{2}
\end{array}\right. \\
& \left\{\begin{array}{l}
\phi_{0}=\tan ^{-1}\left(\frac{X_{1}}{z_{1}-z}\right) \\
\phi_{u}=\tan ^{-1}\left(\frac{X_{2}}{z_{2}-z}\right),
\end{array}\right.
\end{aligned}
$$

and

$$
\left\{\begin{array}{l}
X_{1}=X+\left(z_{1}-z\right) \tan \theta \\
X_{2}=X+\left(z_{2}-z\right) \tan \theta
\end{array},\right.
$$

expressions of $\partial g / \partial x$ and $\partial g / \partial z$ can be derived:

$$
\begin{aligned}
\frac{\partial g(x, z)}{\partial x}= & G \rho\left\{\left[\phi_{u} \sin 2 \theta+\left(2\left(z_{2}-z\right)+X \sin 2 \theta\right)\right.\right. \\
& \left.\times\left(\frac{z_{2}-z}{R_{u}^{2}}\right)\right]-\left[\phi_{0} \sin 2 \theta+\left(2\left(z_{1}-z\right)\right.\right. \\
& \left.+X \sin 2 \theta)\left(\frac{z_{1}-z}{R_{0}^{2}}\right)\right]+\cos ^{2} \theta \ln \left(\frac{R_{u}^{2}}{R_{0}^{2}}\right) \\
& \left.+X \cos ^{2} \theta\left(\frac{2 X_{2}}{R_{u}^{2}}-\frac{2 X_{1}}{R_{0}^{2}}\right)\right\} .
\end{aligned}
$$

This simplifies to

$$
\frac{\partial g(x, z)}{\partial x}=G \rho\left[2\left(\phi_{u}-\phi_{0}\right) \sin 2 \theta+\cos ^{2} \theta \ln \left(\frac{R_{u}^{2}}{R_{0}^{2}}\right)\right]
$$

and

$$
\begin{aligned}
\frac{\partial g(x, z)}{\partial z}= & G \rho\left\{\left[-2 \cos ^{2} \theta \phi_{u}+\left(2\left(z_{2}-z\right)\right.\right.\right. \\
& \left.+X \sin 2 \theta)\left(\frac{X_{2}}{R_{u}^{2}}\right)\right]-\left[-2 \cos ^{2} \theta \phi_{0}\right. \\
& \left.+\left(2\left(z_{1}-z\right)+X \sin 2 \theta\right)\left(\frac{X_{1}}{R_{0}^{2}}\right)\right] \\
& +\sin \theta \cos \theta \ln \left(\frac{R_{u}^{2}}{R_{0}^{2}}\right) \\
& \left.+2 X \cos ^{2} \theta\left(\frac{z_{1}-z}{R_{0}^{2}}-\frac{z_{2}-z}{R_{u}^{2}}\right)\right\} .
\end{aligned}
$$

After simplification,

$$
\frac{\partial g(x, z)}{\partial z}=G \rho\left[2\left(\phi_{0}-\phi_{u}\right) \cos ^{2} \theta+\sin \theta \cos \theta \ln \left(\frac{R_{u}^{2}}{R_{0}^{2}}\right)\right] .
$$

\section{Analytic signal and associated complex wavelet coefficients for inclined 2-D gravity step}

Using the notation $a=-z>0$ for the altitudes (also the dilations, positive in the upper half-plane), we define $\mathcal{W}_{\psi_{c}^{1} \lg _{z}(., z=0)}(x, a)$, the $\gamma=1$ complex wavelet, as the analytic signal at all levels above the level of measurement:

$$
\frac{\mathcal{W}_{\psi_{c}^{1} \mid g_{z}(., z)}}{a}=\left(\frac{\partial}{\partial x}+i \frac{\partial}{\partial z}\right) g
$$

That is,

$$
\begin{gathered}
\mathcal{W}_{\psi_{c}^{1} \mid g_{z}(., z)}=G \rho a\left\{\left(\phi_{u}-\phi_{0}\right) \sin 2 \theta+\cos ^{2} \theta \ln \left(\frac{R_{u}^{2}}{R_{0}^{2}}\right)\right. \\
\left.+i\left[2\left(\phi_{0}-\phi_{u}\right) \cos ^{2} \theta+\sin \theta \cos \theta \ln \left(\frac{R_{u}^{2}}{R_{0}^{2}}\right)\right]\right\} .
\end{gathered}
$$


The associated modulus is

$$
\begin{aligned}
& \left|\mathcal{W}_{\psi_{c}^{1} \mid g_{z}(., z)}(x, a)\right| \\
& =G \rho a\left\{\left[\left(\phi_{u}-\phi_{0}\right) \sin 2 \theta+\cos ^{2} \theta \ln \left(\frac{R_{u}^{2}}{R_{0}^{2}}\right)\right]^{2}\right. \\
& \left.\quad+\left[2\left(\phi_{0}-\phi_{u}\right) \cos ^{2} \theta+\cos \theta \sin \theta \ln \left(\frac{R_{u}^{2}}{R_{0}^{2}}\right)\right]^{2}\right\}^{1 / 2}
\end{aligned}
$$

and phase is

$$
\begin{gathered}
\Phi_{\psi_{c}^{1} \mid g_{z}(., z)}(x, a)=\tan ^{-1} \\
\left(\frac{2\left(\phi_{0}-\phi_{u}\right) \cos ^{2} \beta+\cos \theta \sin \theta \ln \left(\frac{R_{u}^{2}}{R_{0}^{2}}\right)}{\left(\phi_{u}-\phi_{0}\right) \sin 2 \theta+\cos ^{2} \theta \ln \left(\frac{R_{u}^{2}}{R_{0}^{2}}\right)}\right) .
\end{gathered}
$$

\section{APPROXIMATED MODULUS OF THE $\mathcal{W}_{\psi_{c}^{1} \mid g_{z}(,, z)}$ WAVELET ALONG THE MAXIMA LINE AT LARGE DILATIONS-GRAVITY CASE OF THE INCLINED 2-D STEP}

In the case of the inclined 2-D step, the modulus maxima line of the $\gamma=1$ complex wavelet transform is curved (see Figure 3). For low dilations $\left(z_{0}+a \ll h / 2\right)$, it points toward the upper edge of the step. For high dilations $\left(z_{0}+a \gg h / 2\right)$, it is a branch of hyperbola whose asymptote points toward the barycenter of the step $(X=0)$. We want to derive analytically the form of this hyperbola. Then we show how this can be used, in an inversion scheme, to recover simultaneously the average depth, shape, and thickness of the inclined step.

Along the maxima line (for $X=0$ ), we have

$$
\left\{\begin{array}{l}
R_{0}^{2}=\left(\frac{h}{2} \tan \theta\right)^{2}+\left(z_{2}+a\right)^{2} \\
R_{u}^{2}=\left(\frac{h}{2} \tan \theta\right)^{2}+\left(z_{1}+a\right)^{2}
\end{array}\right.
$$

and

$$
\left\{\begin{array}{l}
\phi_{0}=\tan ^{-1}\left[\frac{\left(\frac{h}{2}\right) \tan \theta}{z_{1}+a}\right] \\
\phi_{u}=\tan ^{-1}\left[\frac{\left(\frac{h}{2}\right) \tan \theta}{z_{2}+a}\right] .
\end{array}\right.
$$

It follows from equation (A-8) that

$$
\begin{aligned}
& \left|\mathcal{W}_{\psi_{c}^{1} \mid g_{z}(., z)}(x, a)\right| \\
& =G \rho a \cos \theta\left\{4 \left[\tan ^{-1}\left(\frac{h / 2 \tan \theta}{\left(z_{0}+a\right)+h / 2}\right)\right.\right. \\
& \left.\quad-\tan ^{-1}\left(\frac{(h / 2 \tan \theta}{\left(z_{0}+a\right)-h / 2}\right)\right]^{2} \\
& \left.+\ln \left[\frac{(h / 2 \tan \theta)^{2}+\left[\left(z_{0}+a\right)+h / 2\right]^{2}}{(h / 2 \tan \theta)^{2}+\left[\left(z_{0}+a\right)-h / 2\right]^{2}}\right]^{2}\right\}^{1 / 2} .
\end{aligned}
$$

When $z_{0}+a \gg h / 2$, we introduce the infinitesimal dimensionless quantity $\epsilon=(h / 2) /\left(z_{0}+a\right)$. Using a Taylor expansion yields

$$
\begin{aligned}
& \tan ^{-1}\left(\frac{\epsilon \tan \theta}{1+\epsilon}\right)-\tan ^{-1}\left(\frac{\epsilon \tan \theta}{1-\epsilon}\right) \\
& =-2 \epsilon^{2} \tan \theta\left[1-\left(\tan ^{2} \theta-1\right) \epsilon^{2}\right]+O\left(\epsilon^{5}\right)
\end{aligned}
$$

and

$$
\begin{aligned}
& \ln \left[\frac{(\epsilon \tan \theta)^{2}+[1+\epsilon]^{2}}{(\epsilon \tan \theta)^{2}+[1-\epsilon]^{2}}\right] \\
& =4 \epsilon\left[1+\epsilon^{2}\left[\frac{4}{3}-\left(\tan ^{2} \theta+1\right)\right]\right]+O\left(\epsilon^{5}\right) .
\end{aligned}
$$

Finally comes the modulus of $\mathcal{W}_{\psi_{c}^{1} \mid g z(., z)}$ along the maxima line for high dilations $\left(z_{0}+a \gg h / 2\right)$ :

$$
\begin{aligned}
& \left|\mathcal{W}_{\psi_{c}^{1} \mid g_{z}(., z=0)}(X=0, a)\right| \\
& =4 G \rho a \cos \theta\left(\frac{h / 2}{z_{0}+a}\right)\left[1+\frac{1}{3}\left(\frac{h / 2}{z_{0}+a}\right)^{2}\right] \\
& \quad+O\left(\frac{h / 2}{z_{0}+a}\right)^{5} .
\end{aligned}
$$

Using the log notation,

$$
\begin{aligned}
& \ln \left[\frac{\left|\mathcal{W}_{\psi_{c}^{1} \mid g_{z}(., z=0)}(X=0, a)\right|}{a}\right] \\
& \simeq\left\{\ln (2 G \rho a \cos \theta)+\beta \ln \left(z_{0}+a\right)\right\} \\
& \quad+\left\{\ln \left[1+\frac{1}{2}\left(\frac{2}{3}-\tan ^{2} \theta\right)\left(\frac{h / 2}{z_{0}+a}\right)^{2}\right]\right\} .
\end{aligned}
$$

The first-order term corresponds to a local source [refer to equation (14)], where the power law exponent $\beta$ is equal to -1 . The second-order term is a residual $R$ that corresponds to the extension of the step such that

$$
e^{R}-1 \simeq \frac{1}{2}\left(\frac{2}{3}-\tan ^{2} \theta\right)\left(\frac{h / 2}{z_{0}+a}\right)^{2} .
$$

This allows for an estimator $H(a)$ of the thickness $h$ of the step at large dilations. That is, $\lim _{a \rightarrow \infty} H(a)=h$, with

$$
H(a) \simeq 2\left(z_{0}+a\right)\left(\frac{\sqrt{2}}{\sqrt{2 / 3-\tan ^{2} \theta}}\right) \sqrt{e^{R}-1} .
$$

